\title{
Nonparametric Engel Curves and Revealed Preference
}

Blundell, Richard; Browning, Martin; Crawford, Ian

Publication date:

2002

Document version

Publisher's PDF, also known as Version of record

Citation for published version (APA):

Blundell, R., Browning, M., \& Crawford, I. (2002). Nonparametric Engel Curves and Revealed Preference. Institute of Economics, University of Copenhagen. 


\section{CAM}

Centre for Applied

Microeconometrics

Institute of Economics

University of Copenhagen

http://www.econ.ku.dk/CAM/

Nonparametric Engel Curves and Revealed Preference

Richard Blundell

Martin Browning

lan Crawford

2002-04

The activities of CAM are financed by a grant from

The Danish National Research Foundation 


\title{
Nonparametric Engel Curves and Revealed Preference
}

\author{
Richard Blundell; Martin Browning ${ }^{\dagger}$ and Ian Crawford ${ }^{\ddagger}$
}

March 2002

\begin{abstract}
This paper applies revealed preference theory to the nonparametric statistical analysis of consumer demand. Knowledge of expansion paths is shown to improve the power of nonparametric tests of revealed preference. The tightest bounds on indifference surfaces and welfare measures are derived using an algorithm for which revealed preference conditions are shown to guarantee convergence. Nonparametric Engel curves are used to estimate expansion paths and provide a stochastic structure within which to examine the consistency of household level data and revealed preference theory. An application is made to a long time series of repeated crosssections from the Family Expenditure Survey for Britain. The consistency of these data with revealed preference theory is examined. For periods of consistency with revealed preference, tight bounds are placed on true cost of living indices.
\end{abstract}

Keywords: Consumer demands, nonparametric regression, revealed preference.

JEL Classification: D12, C14, C43.

${ }^{*}$ University College London, Department of Economics, Gower Street, London, WC1E 6BT and Institute for Fiscal Studies, r.blundell@ucl.ac.uk, http://www.ifs.org.uk

†University of Copenhagen.

${ }^{\ddagger}$ Institute for Fiscal Studies. 


\section{Introduction ${ }^{1}$}

The attraction of revealed preference (RP) theory is that it allows an assessment of the empirical validity of the usual integrability conditions without the need to impose particular functional forms on preferences. Although developed to describe individual demands by Afriat (1973) and Diewert (1973) following the seminal work of Samuelson (1938) and Houthakker (1950), it has usually been applied to aggregate data but this presents a number of problems ${ }^{2}$. First, on aggregate data, 'outward' movements of the budget line are often large enough, and relative price changes are typically small enough, that budget lines rarely cross (see Varian (1982), Bronars (1987) and Russell (1992)). This means that aggregate data may lack power to reject RP conditions. Second, if we do reject RP conditions on aggregate data we have no way of assessing whether this is due to a failure at the micro level or to the inappropriate aggregation across households that do satisfy the integrability conditions but who have different non-homothetic preferences. By combining nonparametric statistical methods with a revealed preference analysis of micro data we can overcome the problems we have described.

We also have a number of other motivations for this study. First, parametric demand studies on micro data often reject Slutsky symmetry which is one of the implications of utility maximisation subject to a linear budget constraint. Amongst the many possible explanations for this rejection are that either we have the 'wrong' functional form or that there exists no well-behaved form of preferences which can rationalise the data.

\footnotetext{
${ }^{1}$ We are grateful to James Banks, Laura Blow, Tom Crossley, Alan Duncan, Jin Hahn, Hide Ichimura, Arthur Lewbel, Ian Preston, the co-editor and three anonymous referees as well as seminar participants in Berkeley, Bonn, Bristol, Chicago, CREST, Copenhagen, Havard-MIT, Northwestern, Iowa, University of British Columbia and University College Dublin for helpful comments. This study is part of the program of research of the ESRC Centre for the Microeconomic Analysis of Fiscal Policy at IFS. The financial support of the ESRC, the Danish SSF and the Danish National Research Council through its grant to CAM is gratefully acknowledged. Material from the FES made available by the ONS through the ESRC Data Archive has been used by permission of the controller of HMSO. Neither the ONS nor the ESRC Data Archive bear responsibility for the analysis or the interpretation of the data reported here. The usual disclaimer applies.

${ }^{2}$ See Manser and McDonald (1988), and references therein.
} 
Nonparametric analysis allows us to check this. Second, it has proven difficult to test for (global) negative semi-definiteness of the Slutsky matrix in parametric demand models. Using nonparametric revealed preference analysis we can simultaneously test for both symmetry and negative semi-definiteness. Third, if the integrability conditions are not rejected, we often wish to go on and use demand estimates for policy analysis. Using parametric analysis there is always some uncertainty as to how much the welfare conclusions are driven by functional form. If we employ nonparametric techniques then we can obtain bounds on welfare effects and use these bounds to judge the importance of the choice of functional form on welfare conclusions. Fourth, the nonparametric analysis can aid in the development of new and parsimonious parametric demand systems. Finally, we can extend the nonparametric analysis to investigate revealed preference for conditional demands.

The layout of the paper is as follows. In Section 2 we present the specifics of testing the Generalised Axiom of Revealed Preference (GARP). We then develop a method for choosing a sequence of total expenditures that maximise the power of tests of GARP with respect to a given preference ordering. We term this the sequential maximum power (SMP) path. We present some simulation evidence that shows that our GARP tests have considerable power against some alternatives, but not others. We then develop a method of bounding true cost of living indices. Algorithms are presented which give 'tightest' upper and lower bounds for indifference curves passing through any chosen point in the commodity space. We also show how these methods can be used to calculate tight bounds on annual inflation rates without making parametric assumptions.

Section 3 presents a framework for implementing our procedures by using nonparametric Engel curves for each commodity. To do this we assume that households in the same time period and location face the same relative prices. Under this assumption, the nonparametric Engel curves correspond to expansion paths for each price regime. In estimation we address two key issues that arise when placing local average demands in 
a structural economic context. First, we consider the problem of pooling nonparametric Engel curves across households of different demographic composition. We show that a partially linear model that allows for demographic variation (see, for example, Robinson (1988)) has the very unattractive property that it reduces to Piglog demands (budget shares are linear in log total outlay) under homogeneity and symmetry. We then show that the shape invariant model of Härdle and Marron (1990) provides a theory consistent generalisation to the partially linear semiparametric method of pooling nonparametric Engel curves across households of different composition. Second, we allow for the endogeneity of log total expenditure in the nonparametric budget share equations. This section concludes with a discussion of the issues surrounding unobservable preference heterogeneity ${ }^{3}$. We evaluate the use of local average demands in the presence of unobserved heterogeneity and derive a measure of the bias that results in measuring the welfare cost of finite price changes.

In Section 4 we present an empirical investigation of revealed preference using British Family Expenditure Survey data from 1974 to 1993. This long time series of crosssections is used to estimate the associated nonparametric Engel curves for 22 goods, adjusted for endogeneity and demographic composition. We then examine whether revealed preference theory can be rejected for particular sub-periods of the data. From the asymptotic distribution theory for nonparametric regression we are able to provide a statistical structure within which to examine the consistency of data with revealed preference theory without imposing a global parametric structure to preferences. The approach we adopt provides an alternative to the Afriat inefficiency measure explored in Famulari (1995) and Mattei (1994). We find that GARP is not rejected for long periods of our data for most income groups. We also compute bounds for a true cost of living index over the period and annual inflation rates. We compare these bounds to

\footnotetext{
${ }^{3}$ Even taking a small number of households in different price regimes usually leads to a rejection of the nonparametric conditions (see Koo (1963), Mossin (1972) and Mattei (1994), for example, and the recent paper by Sippel (1997) on the use of experimental data).
} 
popular price index numbers and to other nonparametric bounds. The new bounds we derive are shown to provide considerable improvements on classical revealed preference bounds. Section 6 concludes with a summary of our results and a consideration of future directions.

\section{Individual Data and Revealed Preference}

\subsection{Revealed Preference and Observed Demands}

Suppose we wished to test experimentally whether a particular agent had 'rational' and stable preferences. In the context of demand, we could do this by facing the agent with a series of prices and total expenditures and testing whether their demand responses satisfy the Slutsky conditions. Specifically, suppose we have $T$ periods, $t=1 \ldots T$, and we choose $J$-vectors of (positive) prices $\mathbf{p}_{t}$ and (positive) total expenditures $x_{t}$ for each period. We assume that every agent responds with a unique positive demand for each price vector and outlay:

Assumption 1. For each agent there exists a set of demand functions $\mathbf{q}(\mathbf{p}, x): \Re_{++}^{J+1} \rightarrow$ $\Re_{++}^{J}$ which satisfy adding-up: $\mathbf{p}^{\prime} \mathbf{q}(\mathbf{p}, x)=x$ for all prices $\mathbf{p}$ and total outlays $x$.

Thus we are implicitly assuming that preferences are strictly convex and locally non-satiated (but not necessarily transitive). For a given price vector $\mathbf{p}_{t}$ we denote the corresponding $J$-valued function of $x$ as $\mathbf{q}_{t}(x)$ (with $q_{t}^{j}(x)$ for good $j$ ) which we shall refer to as an expansion path for the given prices. We shall also have need of the following assumption:

Assumption 2. Weak normality: if $x>x^{\prime}$ then $q_{t}^{j}(x) \geq q_{t}^{j}\left(x^{\prime}\right)$ for all $j$ and all $\mathbf{p}_{t}$.

Thus increasing total outlay does not lead to a reduced demand for any good. Adding up and weak normality imply that at least one of the inequalities in this assumption is strict and that expansion paths are continuous.

For our hypothetical experiment we could observe the demands for the given prices 
and total outlays and test whether the resulting series of prices and demands satisfy revealed preference tests. To do this we need to define a variety of revealed preference relationships. We say that $\mathbf{q}_{t}\left(x_{t}\right)$ is directly revealed weakly preferred to $\mathbf{q}^{*}$ if the latter is affordable at period $t$ prices and total expenditure $x_{t}: \mathbf{p}_{t}^{\prime} \mathbf{q}_{t}\left(x_{t}\right) \geq \mathbf{p}_{t}^{\prime} \mathbf{q}^{*}$ which we write as $\mathbf{q}_{t}\left(x_{t}\right) R^{0} \mathbf{q}^{*}$. An alternative characterisation is that $\mathbf{q}^{*}$ is within the budget set defined by $\left(\mathbf{p}_{t}, x_{t}\right)$. If the inequality in this condition is strict then we say that $\mathbf{q}_{t}\left(x_{t}\right)$ is directly revealed strictly preferred to $\mathbf{q}^{*}\left(\mathbf{q}_{t}\left(x_{t}\right) P^{0} \mathbf{q}^{*}\right)$ since the agent could have obtained the latter more cheaply (at the prices $\mathbf{p}_{t}$ ) but chose not to. In this case, of course, $\mathbf{q}^{*}$ is in the interior of the budget set defined by $\left(\mathbf{p}_{t}, x_{t}\right)$.

Now consider any sequence of prices and total outlays $\left\{\mathbf{p}_{s}, \mathbf{p}_{t}, \mathbf{p}_{u}, \ldots \mathbf{p}_{v}, \mathbf{p}_{w} ; x_{s}, x_{t}, x_{u}, \ldots x_{v}, x_{w}\right\}{ }^{4}$ We say that the sequence of associated demand vectors $\left\{\mathbf{q}_{s}\left(x_{s}\right), \mathbf{q}_{t}\left(x_{t}\right), \mathbf{q}_{u}\left(x_{u}\right), \ldots \mathbf{q}_{v}\left(x_{v}\right), \mathbf{q}_{w}\left(x_{w}\right)\right\}$ is preference ordered if $\mathbf{q}_{s}\left(x_{s}\right) R^{0} \mathbf{q}_{t}\left(x_{t}\right), \mathbf{q}_{t}\left(x_{t}\right) R^{0} \mathbf{q}_{u}\left(x_{u}\right), \ldots \quad \mathbf{q}_{v}\left(x_{v}\right) R^{0} \mathbf{q}_{w}\left(x_{w}\right)$. Thus a sequence of demands is preference ordered if each demand is directly revealed at least as good as the next one. Given this, we say that $\mathbf{q}_{s}\left(x_{s}\right)$ is revealed weakly preferred to $\mathbf{q}_{w}\left(x_{w}\right)$ if there is a preference ordered sequence starting at the former and ending at the latter; we denote this by $\mathbf{q}_{s}\left(x_{s}\right) R \mathbf{q}_{w}\left(x_{w}\right)$. Suppose now that we have $\mathbf{q}_{s}\left(x_{s}\right) R \mathbf{q}_{w}\left(x_{w}\right)$ and that we also have that the final demand in the sub-sequence, $\mathbf{q}_{w}\left(x_{w}\right)$, is directly revealed strictly preferred to the first demand vector $\mathbf{q}_{s}\left(x_{s}\right)$ (that is, $\left.\mathbf{q}_{w}\left(x_{w}\right) P^{0} \mathbf{q}_{s}\left(x_{s}\right)\right)$. In this case we say that this sub-sequence fails GARP, the general axiom of revealed preference. We shall say that a set of prices and demands fails GARP if any sub-sequence drawn from the set fails GARP. To illustrate, suppose that we have five time periods and that $\mathbf{q}_{4}\left(x_{4}\right) R^{0} \mathbf{q}_{2}\left(x_{2}\right), \mathbf{q}_{2}\left(x_{2}\right) R^{0} \mathbf{q}_{1}\left(x_{1}\right)$ and $\mathbf{q}_{1}\left(x_{1}\right) P^{0} \mathbf{q}_{4}\left(x_{4}\right)$. Thus the sub-sequence $\left\{\mathbf{q}_{4}\left(x_{4}\right), \mathbf{q}_{2}\left(x_{2}\right), \mathbf{q}_{1}\left(x_{1}\right)\right\}$ fails GARP ${ }^{5}$ and consequently the set $\left(\mathbf{q}_{1}\left(x_{1}\right), \mathbf{q}_{2}\left(x_{2}\right), \mathbf{q}_{3}\left(x_{3}\right), \mathbf{q}_{4}\left(x_{4}\right), \mathbf{q}_{5}\left(x_{5}\right)\right)$ fails GARP.

\footnotetext{
${ }^{4}$ We remind the reader that the order matters for a sequence (so that $\{1,2,3\}$ is different from $\{3,1,2\})$ but not for sets (so that the sets $(1,2,3)$ and $(2,3,1)$ are the same).

${ }^{5}$ Note that this does not necesarily imply that the sub-sequence $\left\{\mathbf{q}_{1}\left(x_{1}\right), \mathbf{q}_{4}\left(x_{4}\right), \mathbf{q}_{2}\left(x_{2}\right)\right\}$ fails GARP.
} 


\subsection{Choosing a Path for Comparison Points}

Below we take the sequence of (absolute) prices $\left\{\mathbf{p}_{1}, \mathbf{p}_{2}, \ldots \mathbf{p}_{T}\right\}$ that is given by our data set but we are free to choose the sequence of total expenditures used in the comparisons above. When considering how to do this, there is a well known problem with applying GARP tests to data to which Varian (1982) refers in his applied work. This problem arises since, particularly with time series data, income growth over time can swamp variations in relative prices (which are what we are interested in). This is because real income growth induces outward movements of the budget constraint and, combined with typically small period-to-period relative price movements, this means that budget lines may seldom cross. As a result, data often lacks power to reject GARP. Indeed, if we choose the $x_{t}$ 's so that budget lines never cross then we can never violate the GARP conditions. Clearly then, with a given set of relative prices the power of a revealed preference test will depend critically on the choice of the outlay path $\left(x_{1}, x_{2}, \ldots x_{T}\right)$.

One possible solution is to choose a sequence of constant 'real' total expenditures. Thus given $x_{1}$ and a set of price indices $\left(P_{1}\left(\mathbf{p}_{1}\right), P_{2}\left(\mathbf{p}_{2}\right), \ldots P_{T}\left(\mathbf{p}_{T}\right)\right)$ we could choose $x_{t}=$ $x_{1} P_{t} / P_{1}$. Although superficially attractive this begs the question of what price index to use. More importantly, even if the series of demands generated in this way did satisfy GARP, we cannot be sure that any other series of total expenditures 'starting' from $x_{1}$ would also satisfy GARP. Instead of this, we present an algorithm for determining a sequence of demands which maximises the chance of finding a rejection given a particular preference ordering of the data.

Consider any sub-sequence (taken to be of length 5 for illustrative purposes) of prices $\left\{\mathbf{p}_{s}, \mathbf{p}_{t}, \mathbf{p}_{u}, \mathbf{p}_{v}, \mathbf{p}_{w}\right\}$. Now take an outlay $x_{u}$ in period $u$ with associated demand $\mathbf{q}_{u}\left(x_{u}\right)$. We can construct a preference ordered sequence through $\mathbf{q}_{u}\left(x_{u}\right)$ for this sequence of prices by using two recursive schemes, one forwards and the other backwards. For the backwards part (the set of demands that are at least as good as $\mathbf{q}_{u}\left(x_{u}\right)$ ) we set total 
Figure 2.1: Testing GARP with expansion paths

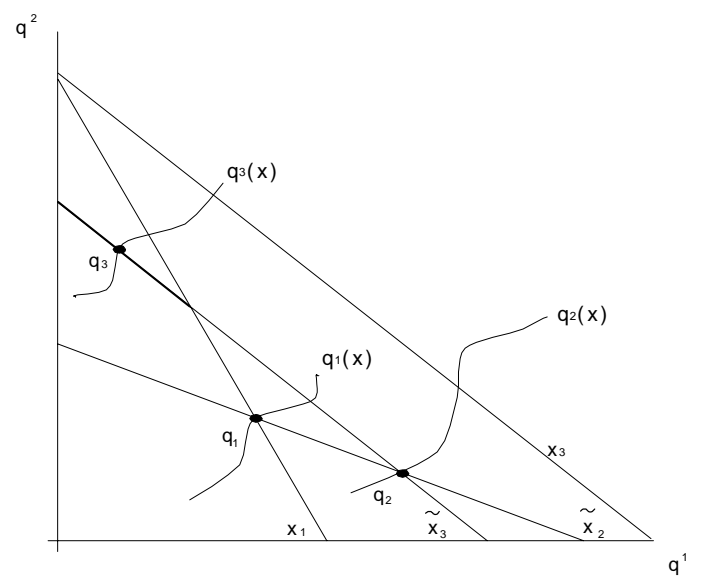

outlay in period $t$ so that the period $u$ quantity bundle is just affordable: $\tilde{x}_{t}=\mathbf{p}_{t}^{\prime} \mathbf{q}_{u}\left(x_{u}\right)$. Thus $\tilde{\mathbf{q}}_{t}=\mathbf{q}_{t}\left(\tilde{x}_{t}\right)$ is the 'lowest' point on the period $t$ expansion path which is directly revealed at least as good as $\mathbf{q}_{u}\left(x_{u}\right)$. Then set $\tilde{\mathbf{q}}_{s}=\mathbf{q}_{s}\left(\mathbf{p}_{s}^{\prime} \tilde{\mathbf{q}}_{t}\right)$. Thus the sequence $\left\{\tilde{\mathbf{q}}_{s}, \tilde{\mathbf{q}}_{t}, \mathbf{q}_{u}\left(x_{u}\right)\right\}$ is preference ordered.

To construct the path of quantities to which $\mathbf{q}_{u}\left(x_{u}\right)$ is weakly preferred, we first solve for the value of outlay in period $v$ that satisfies $x_{u}=\mathbf{p}_{u}^{\prime} \mathbf{q}_{v}\left(x_{v}\right)$, which we denote $\tilde{x}_{v}$, with demand $\tilde{\mathbf{q}}_{v}=\mathbf{q}_{v}\left(\tilde{x}_{v}\right){ }^{6}$ This is constructed so that $\tilde{\mathbf{q}}_{v}$ is the 'highest' demand on the period $v$ expansion path to which $\mathbf{q}_{u}\left(x_{u}\right)$ is directly revealed weakly preferred. Then construct $\tilde{\mathbf{q}}_{w}=\mathbf{q}_{w}\left(\tilde{x}_{w}\right)$ by setting $\tilde{x}_{v}=\mathbf{p}_{v}^{\prime} \mathbf{q}_{w}\left(\tilde{x}_{w}\right)$. By construction, the entire path $-\left\{\tilde{\mathbf{q}}_{s}, \tilde{\mathbf{q}}_{t}, \mathbf{q}_{u}\left(x_{u}\right), \tilde{\mathbf{q}}_{v}, \tilde{\mathbf{q}}_{w}\right\}$ - is preference ordered. We term the path created in this way a sequential maximum power (SMP) path through $\mathbf{q}_{u}\left(x_{u}\right)$. An SMP path is said to start (respectively, finish) at $\mathbf{q}_{u}\left(x_{u}\right)$ if the latter is the first (respectively, the last)

\footnotetext{
${ }^{6}$ Given continuity and weak normality of the expansion paths there always exists a unique outlay and demand that satisfies this condition.
} 
element in the sequence. Although we do not denote it explicitly it is important to recognise that an SMP path is always defined relative to a sequence of time indices (in this illustration $\{s, t, u, v, w\})$ and a point on an expansion path for one of these time periods (in this case, $\mathbf{q}_{u}\left(x_{u}\right)$ ). For example, $\left\{\tilde{\mathbf{q}}_{s}, \tilde{\mathbf{q}}_{t}, \mathbf{q}_{u}\left(x_{u}\right)\right\}$ is an SMP path finishing at $\mathbf{q}_{u}\left(x_{u}\right)$.

To illustrate why this gives maximal power for a particular sequence, consider the three period, two good example in figure 2.1. Here the order of the sequence is $\{3,2,1\}$ finishing at $\mathbf{q}_{1}\left(x_{1}\right)$ so that $\left\{\mathbf{q}_{3}\left(\tilde{x}_{3}\right) R^{0} \mathbf{q}_{2}\left(\tilde{x}_{2}\right) R^{0} \mathbf{q}_{1}\left(x_{1}\right)\right\}$. In this figure the shaded part of the period 3 budget line gives the demands which result in a rejection of GARP. One can see that if we took any other preference ordered path of demands with the same sequence $\left(\mathbf{q}_{3}\left(x_{3}\right) R^{0} \mathbf{q}_{2}\left(x_{2}\right) R^{0} \mathbf{q}_{1}\left(x_{1}\right)\right)$ this would reduce the length of this segment. This is because any such path pushes out the period 3 budget line which reduces the chance of observing a GARP rejecting demand in period 3 (if demands are weakly normal). ${ }^{7}$ More formally, we have:

Proposition 1. Suppose that the demand sequence

$$
\left\{\mathbf{q}_{s}\left(x_{s}\right), \mathbf{q}_{t}\left(x_{t}\right), \mathbf{q}_{u}\left(x_{u}\right) \ldots, \mathbf{q}_{v}\left(x_{v}\right), \mathbf{q}_{w}\left(x_{w}\right)\right\}
$$

rejects GARP. If demands are weakly normal then the SMP path for the same sequence of periods ending at $\mathbf{q}_{w}\left(x_{w}\right)$ :

$$
\left\{\mathbf{q}_{s}\left(\tilde{x}_{s}\right), \mathbf{q}_{t}\left(\tilde{x}_{t}\right), \mathbf{q}_{u}\left(\tilde{x}_{u}\right) \ldots, \mathbf{q}_{v}\left(\tilde{x}_{v}\right), \mathbf{q}_{w}\left(x_{w}\right)\right\}
$$

also rejects $G A R P$.

Proof. See Appendix A.

Thus, if we test for GARP along a given SMP path finishing at $\mathbf{q}_{w}\left(x_{w}\right)$ and we do not reject, then we can be confident that we would not reject for any other preference ordered path which finishes at the same demand and maintains the preference ordering implied by the SMP path. It is important to note that there may be other preference

\footnotetext{
${ }^{7}$ This is valid for the true expansion path. In our empirical work below we use estimated expansion paths. For these, there is the possibility that the precision of the estimated path is such that although the length is reduced the probablility of rejection is not.
} 
orderings that finish at $\mathbf{q}_{w}\left(x_{w}\right)$ that do reject GARP so that our maximal power is always with respect to a particular sequencing of time periods. In our empirical work below we always take the chronological sequence finishing in period 1. It is important also to note that maintaining the ordering of demands but choosing a different end point $-\mathbf{q}_{w}\left(x_{w}^{\prime}\right)$ instead of $\mathbf{q}_{w}\left(x_{w}\right)$ - will result in a different SMP path which may violate GARP, even if the SMP path finishing at $\mathbf{q}_{w}\left(x_{w}\right)$ does not. This is easiest to see in the two good, two expansion path case. Suppose, for example, expansion paths are such that GARP is not rejected if we take an SMP path finishing at a low level of expenditure for one of the demands. If the expansion paths cross and we consider an SMP path finishing at an expenditure level above the crossing point, then we will reject GARP on that path. To check this we take a number of quantile points in the $x$ distribution and apply the SMP procedure to demand sequences ending at $\mathbf{q}_{w}(x)$ where $\mathbf{q}_{w}(x)$ is evaluated at each of these outlays.

\subsection{The Power of Parametric and Revealed Preference Tests of Integrability.}

When considering tests of integrability, whether parametric or nonparametric, we must be careful to recognise that there are some alternatives against which both modes of test will have low power. To illustrate with a well known example, suppose we draw a large independent sample each period from a large population of agents. If each agent in each period chooses demands on their budget surface by drawing from a uniform distribution on the budget surface then in general no individual path of demands will be integrable. However the (population and sample) mean data will appear to be generated by a Cobb-Douglas utility function with weights equal to the inverse of the number of commodities (see Becker (1962) and Grandmont (1992)). Parametric and revealed preference tests are unlikely to reject the integrability conditions for such data but it is not clear that we would wish to characterise them as the outcome of a 'rational' procedure. Equally there will be paths of relative prices which lead to low power tests 
of the integrability conditions under certain alternatives. The extreme case is if we have no variation in relative prices in which case, of course, we cannot estimate price effects for parametric models and we have only one expansion path for our GARP tests.

Thus many of the concerns with the power of tests of the integrability conditions are common to both parametric and revealed preference tests. There is also a concern, however, that revealed preference tests are inherently lacking in power (as compared with parametric tests) and will fail to reject 'too often'. However, we know from the discussion of the previous sub-sections that the nonparametric approach can be used to test Revealed Preference conditions without recourse to any parametric specification of preferences. In the event that they do not reject, parametric models will be able to improve on the bounds that we derive for cost of living and welfare measurement using revealed preference alone. One possible strategy for future work is to go on to consider flexible parametric models over regions where the nonparametric tests do not fail. We emphasise again that one of our concerns regarding currently used parametric models is that they may be too inflexible and in particular they may unduly restrict differences in price effects between rich and poor.

To investigate this issue further we consider three alternative generating processes that produce non-integrable demands: a random procedure, an integrable path with measurement error and a path generated by a slow adjustment model. All of the calculations below use the actual sequence of relative prices observed in our data which is the relevant set of relative prices. For the random alternative we suppose that the demand at any price/income configuration is a draw from a uniform distribution on the budget surface (just as we assumed for individual agents in the illustration above but without the averaging). The SMP procedure with a given sequence of prices is: choose $x_{1}$ and draw the vector $\mathbf{q}_{1}$ from a uniform distribution on the budget surface given by $\left(\mathbf{p}_{1}, x_{1}\right)$. Then set $\hat{x}_{2}=\mathbf{p}_{2}^{\prime} \mathbf{q}_{1}$ and draw $\hat{\mathbf{q}}_{2}$ from a uniform distribution on the new budget surface. Continue for all $T$ periods. We can show analytically that if we have 
only two periods and two goods then GARP will only reject half of the time. This indicates low power. On the other hand, as the number of periods grows the probability of rejecting grows. The actual rejection probability depends on the number of periods and the relative price variability. To illustrate this we take the actual sequence of relative prices we have in our data (for 22 goods over 20 years; details are given in the empirical section below) and generate demands according to this alternative. We found that in 10,000 random simulated SMP paths we reject GARP every time. This indicates that our procedure does have considerable power against this particular alternative. However, a sceptic might argue that any procedure that failed to reject the rationality of such an unstructured alternative would be very poor indeed. Thus we also consider two other alternatives which are 'close' to integrable.

Our second procedure is to take a set of demands that are integrable and to incorporate a multiplicative measurement error. ${ }^{8}$ Specifically, in each period we draw a $(22 \times 1)$ vector of budget shares from a joint distribution in which each budget share has a fixed mean approximately ${ }^{9}$ equal to the average budget shares in 1974; see Appendix C for details. This is equivalent to taking a Cobb-Douglas utility function over 22 goods with fixed budget shares and then multiplying by a unit mean measurement error. Clearly, if we set the variance of the budget share draws in this procedure to zero then we have a path of Cobb-Douglas demands which satisfy GARP. Conversely, if we allow for a great deal of measurement error then we shall almost certainly reject GARP. The critical issue, then is how much measurement error is it reasonable to allow for? We calibrate this to the variance of the budget shares in our data which gives an upper bound on measurement error. We then take different proportions of these variances and simulate

\footnotetext{
${ }^{8}$ An alternative interpretation is that for each price regime we generate a sample which is an independent draw from the same population with a given distribution of heterogeneity over the preference parameters.

${ }^{9}$ Adding-up implies that we need to normalise by the sum of random variables across all shares. Consequently, the mean of the simulated errors on the budget shares will involve the mean of the ratio of random varaibles. In our simulations this ratio has a mean close to unity.
} 
10,000 times and record the proportion of rejections. We find that even very modest amounts of noise cause rejection. For example, if we allow for only $0.5 \%$ (respectively $1 \%$ and $2 \%$ ) of the total variance to be due to noise and use these in our simulations then we reject $61 \%$, (respectively, $87 \%$ and $97 \%$ ) of the time. Thus the GARP/SMP procedure has considerable power against this alternative.

The third demand generating process we consider is a 'naive' adjustment model. In this we assume that households adjust slowly to the optimum for the prices in that period. Specifically, if we take (integrable) demands $\mathbf{q}(\mathbf{p}, x)$, we set the period $t$ demand $\tilde{\mathbf{q}}\left(\mathbf{p}_{t}, x_{t}\right)$ to:

$$
\tilde{\mathbf{q}}\left(\mathbf{p}_{t}, x_{t}\right)=\lambda \mathbf{q}\left(\mathbf{p}_{t}, x_{t}\right)+(1-\lambda) \tilde{\mathbf{q}}\left(\mathbf{p}_{t-1}, x_{t-1}\right) \text { for } t=2, \ldots T
$$

Thus the sequence of demands will be integrable if we set $\lambda=1$ but as adjustment becomes slower, the likelihood of rejecting GARP increases. Note that this system satisfies 'long run' integrability. For the demand functions we use a Quadratic Almost Ideal System (QUAIDS) (see Banks et al (1997)) with parameters estimated on our sample and the homogeneity and symmetry conditions imposed ${ }^{10}$. For the first period demands at a given outlay $x_{1}$ we set $\mathbf{q}_{1}=\mathbf{q}\left(\mathbf{p}_{1}, x_{1}\right)$ and, as before, subsequent total expenditures are chosen using the SMP path for our price data. Doing this, we find that for the path starting at median first period total outlay, we reject GARP if and only if $\lambda<0.26$. Such a low figure suggests that our testing procedure is unlikely to have good power against an alternative that satisfies the integrability conditions in the long run. Once again, we emphasise that the same may be true of alternative parametric procedures.

\footnotetext{
${ }^{10}$ We do not impose the negativity conditions on our parameter estimates but we note that the 'full adjustment' paths generated by our simulations starting at median total expenditure do pass GARP.
} 


\subsection{Computing Tight Bounds on Welfare Measures}

Afriat (1977) showed how revealed preference restrictions can be used to provide information on the curvature of indifference surfaces in commodity space and then used to set bounds on the welfare effects of a price change. This is further developed in Varian (1982) and Manser and McDonald (1988). One problem with applying this procedure to the aggregate data that the latter use is that budget surfaces rarely cross so that the bounds from such data tend to be wide ${ }^{11}$. Knowledge of expansion paths can greatly improve these bounds. Without loss of generality we consider an indifference surface passing through some base bundle $\mathbf{q}_{1}$ on the first expansion path $\mathbf{q}_{1}(x)$. If GARP and weak normality hold then we shall show that we can partition each expansion path, $\mathbf{q}_{t}(x)$, into three distinct segments. First, on any expansion path, there are the demands that can be shown to be weakly revealed preferred to $\mathbf{q}_{1}$. Second, we have the demands that we can show are weakly revealed dominated by $\mathbf{q}_{1}$. Finally there is an intermediate segment with demands that cannot be revealed preference ordered with respect to $\mathbf{q}_{1}$. We then show how knowledge of these segments for each expansion paths allows us to construct tight bounds on the welfare costs of arbitrary price changes from the base price $\mathbf{p}_{1}$.

We first present an algorithm that we claim finds the 'lowest' point on each expansion path such that we can show $\mathbf{q}_{t} R \mathbf{q}_{1}$; we term this the weakly preferred set. We then show that if GARP and weak normality hold then this algorithm converges in a finite number of steps and the weakly preferred set has the claimed property.

Algorithm A.1 Input: a base bundle $\mathbf{q}_{1}$, price vectors $\mathbf{p}_{t}$ and expansion paths $\mathbf{q}_{t}(x)$ for $t=2, . ., T$. Output: $Q_{B}\left(\mathbf{q}_{1}\right)$.

1) Set $W=\left\{\mathbf{q}_{1}, \mathbf{q}_{2}\left(\mathbf{p}_{2}^{\prime} \mathbf{q}_{1}\right), \ldots ., \mathbf{q}_{T}\left(\mathbf{p}_{T}^{\prime} \mathbf{q}_{1}\right)\right\}$

2) Set $F=\left\{\mathbf{q}_{1}, \mathbf{q}_{2}\left(\min _{\mathbf{q}_{t} \in W}\left\{\mathbf{p}_{2}^{\prime} \mathbf{q}_{t}\right\}\right), \ldots, \mathbf{q}_{T}\left(\min _{\mathbf{q}_{t} \in W}\left\{\mathbf{p}_{T}^{\prime} \mathbf{q}_{t}\right\}\right)\right\}$.

3) If $F \equiv W$ then set $Q_{B}\left(\mathbf{q}_{1}\right)=W$ and stop. Else set $W=F$ and go to (2).

\footnotetext{
${ }^{11}$ Varian (1983) and Manser and McDonald (1988) tighten the bounds using a maintained hypothesis of homotheticity, but this is problematic since much empirical evidence suggests that budget shares are not constant with respect to the total budget.
} 
The set $Q_{B}\left(\mathbf{q}_{1}\right)$ has $T$ elements, one for each expansion path; we denote the th element of $Q_{B}\left(\mathbf{q}_{1}\right)$ by $\mathbf{q}_{t}^{B}$. A discussion of this algorithm and the one following and an illustration can be found in Appendix B. We have:

Proposition 2. If GARP and weak normality hold then:

A. algorithm A.1 converges in a finite number of steps.

$B .\left(\mathbf{q}_{t} \geq \mathbf{q}_{t}^{B}\right) \Leftrightarrow\left(\mathbf{q}_{t} R \mathbf{q}_{1}\right)$.

Proof. See Appendix A.

The first part of the proposition assures that the algorithm is feasible (it in fact converges quite quickly in practice). The second part of the proposition verifies that the algorithm identifies the largest set of points on expansion paths that can be shown to be revealed preferred to $\mathbf{q}_{1}$ with the data to hand. ${ }^{12}$

We also have an algorithm that finds the 'highest' point on each expansion path such that $\mathbf{q}_{1}$ can be shown to be revealed preferred to these points.

Algorithm A.2 Input: a base bundle $\mathbf{q}_{1}$ and price vectors $\mathbf{p}_{t}$ and expansion paths $\mathbf{q}_{t}(x)$ for $t=1,2, . ., T$. Output: $Q_{W}\left(\mathbf{q}_{1}\right)$.

1) Set $W=\left\{\mathbf{q}_{1}, \mathbf{q}_{2}\left(x \mid \mathbf{p}_{1}^{\prime} \mathbf{q}_{2}(x)=\mathbf{p}_{1}^{\prime} \mathbf{q}_{1}\right), \ldots ., \mathbf{q}_{T}\left(x \mid \mathbf{p}_{1}^{\prime} \mathbf{q}_{T}(x)=\mathbf{p}_{1}^{\prime} \mathbf{q}_{1}\right)\right\}$

$2)$ Set $F=\left\{\mathbf{q}_{1}, \max _{\mathbf{q}_{t} \in W}\left(\mathbf{q}_{2}: \mathbf{p}_{t}^{\prime} \mathbf{q}_{t}=\mathbf{p}_{t}^{\prime} \mathbf{q}_{2}\right), \ldots \max _{\mathbf{q}_{t} \in W}\left(\mathbf{q}_{T}: \mathbf{p}_{t}^{\prime} \mathbf{q}_{t}=\mathbf{p}_{t}^{\prime} \mathbf{q}_{T}\right)\right\}$.

3) If $F \equiv W$ then set $Q_{W}\left(\mathbf{q}_{1}\right)=W$ and stop. Else set $W=F$ and go to (2).

Denoting the $t$ th element of $Q_{W}\left(\mathbf{q}_{1}\right)$ by $\mathbf{q}_{t}^{W}$ we have the following results for this algorithm:

Proposition 3. If GARP and weak normality hold then:

A. algorithm A.2 converges in a finite number of steps.

$B$. for any $x$ we have $\left(\mathbf{q}_{t}^{W} \geq \mathbf{q}_{t}\right) \Leftrightarrow\left(\mathbf{q}_{1} R \mathbf{q}_{t}\right)$

Proof. See Appendix A.

Finally we can show that for any $t$ we have $\mathbf{q}_{t}^{B} \geq \mathbf{q}_{t}^{W}$ so that the two points divide any expansion path into three connected segments (given weak normality).

\footnotetext{
${ }^{12}$ We could extend the weakly revealed preferred set to the whole commodity space by taking the convex hull of the points in $Q_{B}\left(\mathbf{q}_{1}\right)$ but this is not necessary for the welfare bounds we derive below.
} 
Given the sets $Q_{W}\left(\mathbf{q}_{1}\right)$ and $Q_{B}\left(\mathbf{q}_{1}\right)$ we can derive bounds on the welfare effects of a price change. For example, suppose that we have a reference commodity level $\mathbf{q}_{1}$ (on the expansion path $\mathbf{q}_{1}(x)$ ) and an arbitrary absolute price vector $\mathbf{p}_{z}$. The true cost-of-living index based at $\mathbf{q}_{1}$ is given by:

$$
\frac{c\left(\mathbf{p}_{z}, \mathbf{q}_{1}\right)}{c\left(\mathbf{p}_{1}, \mathbf{q}_{1}\right)}
$$

where $c\left(\mathbf{p}_{z}, \mathbf{q}_{1}\right)$ is the expenditure function giving the cost of attaining a bundle indifferent to $\mathbf{q}_{1}$ at prices $\mathbf{p}_{z}$. Bounds can be placed on this index using the two sets derived above $^{13}$ :

$$
\frac{\min _{\mathbf{q}}\left\{\mathbf{p}_{z}^{\prime} \mathbf{q} \mid \mathbf{q} \in Q_{W}\left(\mathbf{q}_{1}\right)\right\}}{\mathbf{p}_{1}^{\prime} \mathbf{q}_{1}} \leq \frac{c\left(\mathbf{p}_{z}, \mathbf{q}_{1}\right)}{c\left(\mathbf{p}_{1}, \mathbf{q}_{1}\right)} \leq \frac{\min _{\mathbf{q}}\left\{\mathbf{p}_{z}^{\prime} \mathbf{q} \mid \mathbf{q} \in Q_{B}\left(\mathbf{q}_{1}\right)\right\}}{\mathbf{p}_{1}^{\prime} \mathbf{q}_{1}}
$$

In section 5 we use these results together with nonparametric estimates of Engel curves to compute upper and lower bounds on the true fixed welfare base cost-of-living index over the period 1974 to 1993 using British household budget survey data. These are then compared to standard cost-of-living index formulae and to alternative nonparametric and revealed preference bounds.

As well as being interested in fixed welfare base cost-of-living indices which span a period of, perhaps, several years, we are often even more interested in annual inflation rates and with these it is typical to update the welfare base in each period rather than let it get too out of date. For example the inflation rate between the adjacent years $t$ and $t+1$ may be calculated as $\left(c\left(\mathbf{p}_{t+1}, \mathbf{q}_{t}\right) / c\left(\mathbf{p}_{t}, \mathbf{q}_{t}\right)\right)-1$. Bounds can easily be derived by finding the bounds on the indifference curve through $\mathbf{q}_{t}-$ i.e. $Q_{W}\left(\mathbf{q}_{t}\right)$ and $Q_{B}\left(\mathbf{q}_{t}\right)$

\footnotetext{
${ }^{13}$ Note that there is the possibility of corner solutions with respect to the lower bound whereby the new price vector may cause one or more demands to fall to zero. To allow for this in the calculation of the cost-of-living index the lower bound set $Q_{W}\left(\mathbf{q}_{1}\right)$ needs to be augmented in the following way

$$
Q_{W}\left(\mathbf{q}_{1}\right)=\max _{j}\left\{\mathbf{p}_{w}^{\prime} \mathbf{q}_{w} / p_{w}^{j}: \forall \mathbf{q}_{w} \in Q_{W}\left(\mathbf{q}_{1}\right)\right\} \cup Q_{W}\left(\mathbf{q}_{1}\right)
$$
}

See Appendix B for an illustration. 
- , and by applying

$$
\frac{\min _{\mathbf{q}}\left\{\mathbf{p}_{t+1}^{\prime} \mathbf{q} \mid \mathbf{q} \in Q_{W}\left(\mathbf{q}_{t}\right)\right\}}{\mathbf{p}_{t}^{\prime} \mathbf{q}_{t}} \leq \frac{c\left(\mathbf{p}_{t+1}, \mathbf{q}_{t}\right)}{c\left(\mathbf{p}_{t}, \mathbf{q}_{t}\right)} \leq \frac{\min _{\mathbf{q}}\left\{\mathbf{p}_{t+1}^{\prime} \mathbf{q} \mid \mathbf{q} \in Q_{B}\left(\mathbf{q}_{t}\right)\right\}}{\mathbf{p}_{t}^{\prime} \mathbf{q}_{t}}
$$

The inflation rate between $t+1$ and $t+2$ can be measured as $\left(c\left(\mathbf{p}_{t+2}, \mathbf{q}_{t+1}\right) / c\left(\mathbf{p}_{t+1}, \mathbf{q}_{t+1}\right)\right)-1$ and a bound derived in an identical manner. In section 5 we present annual inflation bounds for 1975 to 1993 derived in this way.

\section{Nonparametric Engel Curves}

\subsection{Kernel Estimation of the Budget Share System}

To estimate the expansion paths for each price regime we employ nonparametric regression methods. Let $\left\{\left(\ln x_{i}, w_{i j}\right)\right\}_{i=1}^{n}$ represent a sequence of $n$ household observations on the $\log$ of total expenditure $\ln x_{i}$ and on the $j$ th budget share $w_{i j}$, for each household $i$ facing the same relative prices. For each commodity $j$, budget shares and total outlay are related by the stochastic Engel curve

$$
w_{i j}=g_{j}\left(\ln x_{i}\right)+\varepsilon_{i j}
$$

where we assume that, for each household $i$, the unobservable term $\varepsilon_{i j}$ satisfies

$$
E\left(\varepsilon_{i j} \mid \ln x\right)=0 \text { and } \operatorname{Var}\left(\varepsilon_{i j} \mid \ln x\right)=\sigma_{j}^{2}(\ln x) \forall \text { goods } j=1, \ldots J
$$

so that the nonparametric regression of budget shares on log total expenditure estimates $g_{j}(\ln x) .{ }^{14} \operatorname{In}(3.1)$, if preferences are Piglog ${ }^{15}, g_{j}$ is linear in $\ln x$ for all goods $j=1, \ldots, J$.

In our empirical application we use the following unrestricted Nadaraya-Watson kernel regression estimator

$$
\widehat{g}_{j}(\ln x)=\frac{\widehat{r}_{j}^{h}(\ln x)}{\widehat{f}^{h}(\ln x)} \equiv \widehat{w}_{j}(\ln x)
$$

\footnotetext{
${ }^{14}$ Below we discuss how we allow for the endogeneity of $\ln x$ in the Engel curve regression equation.

${ }^{15}$ See Muellbauer (1975) and the empirical investigations by Working (1943) and Leser (1963). These are the preferences that underly the popular Translog and Almost Ideal demand systems.
} 
in which

$$
\widehat{r}_{j}^{h}(\ln x)=\frac{1}{n} \sum_{l=1}^{n} K_{h}\left(\ln x-\ln x_{l}\right) w_{l j}
$$

and

$$
\widehat{f}^{h}(\ln x)=\frac{1}{n} \sum_{l=1}^{n} K_{h}\left(\ln x-\ln x_{l}\right),
$$

where $h$ is the bandwidth and $K_{h}(\cdot)=h^{-1} K(\cdot / h)$ for some symmetric kernel weight function $K($.) which integrates to one. We assume the bandwidth $h$ satisfies $h \rightarrow 0$ and $n h \rightarrow \infty$ as $n \rightarrow \infty$. Under standard conditions the estimator (3.3) is consistent and asymptotically normal, see Härdle (1990) and Härdle and Linton (1994). Additionally, provided the same bandwidth and kernel are used to estimate each $g_{j}(\ln x)$, adding-up across the share equations will be automatically satisfied for each $\ln x$ and there is no efficiency gain from combining equations. This mirrors the invariance result for SURE systems with identical regressors (see Deaton (1983), for example).

\subsection{Demographic Composition and Semiparametric Estimation}

Household expenditures typically display variation according to demographic composition. A fully nonparametric approach would be to stratify by each distinct household demographic type and estimate each Engel curve by nonparametric regression within each cell. Given that this would result in relatively small sample sizes within each cell, we choose to use a semiparametric specification to pool across household types.

Let $\mathbf{z}_{i}$ represent a vector of discrete household composition variables for each household observation $i$. A simple semiparametric specification would be to assume partial linearity (see Robinson (1988) and Powell (1987))

$$
w_{i j}=g_{j}\left(\ln x_{i}\right)+\mathbf{z}_{i}^{\prime} \gamma_{j}+\varepsilon_{i j}
$$

with

$$
E\left(\varepsilon_{i j} \mid \mathbf{z}_{i}, \ln x_{i}\right)=0 \text { and } \operatorname{Var}\left(\varepsilon_{i j} \mid \mathbf{z}_{i}, \ln x_{i}\right)=\sigma_{j}^{2}\left(\mathbf{z}_{i}, \ln x_{i}\right) .
$$


in which $\gamma_{j}$ represents a finite parameter vector of household composition effects for commodity $j$ and $g_{j}\left(\ln x_{i}\right)$ is some unknown function as in (3.1).

Although the partially linear model (3.6) motivates the semiparametric approach taken in this paper, consideration of the integrability conditions indicate that some modification is required. This is because the additive structure underlying (3.6) together with the Slutsky symmetry conditions

$$
\frac{\partial w_{j}}{\partial \ln p_{k}}+w_{k} \frac{\partial w_{j}}{\partial \ln x}=\frac{\partial w_{k}}{\partial \ln p_{j}}+w_{j} \frac{\partial w_{k}}{\partial \ln x},
$$

requires that $g($.$) be linear.$

Proposition 4. Suppose that budget shares have a form that is additive in functions of $\ln x$ and demographics

$$
w_{j}(\ln \mathbf{p}, \ln x, \mathbf{z})=m_{j}(\ln \mathbf{p}, \mathbf{z})+g_{j}(\ln \mathbf{p}, \ln x)
$$

If (i) Slutsky symmetry (3.8) holds and (ii) the effects of demographics on budget shares are unrestricted in the sense that $m_{j}$ can vary in any way with $\mathbf{z}$ then $g_{j}($.$) is linear in$ $\ln x$ :

Proof. See Appendix A.

This proposition demonstrates that the additive form given in (3.9) will only be consistent with utility maximisation if we restrict the way in which demographics affect budget shares, or if preferences are Piglog. That is $g_{j}(\ln x)$ is linear in $\ln x$ for all $j$.

An alternative specification that we adopt which does not impose restrictions on the form of $g_{j}$, is the following extension of the partially linear model

$$
w_{i j}=g_{j}\left(\ln x_{i}-\phi\left(\mathbf{z}_{i}^{\prime} \boldsymbol{\theta}\right)\right)+\mathbf{z}_{i}^{\prime} \boldsymbol{\alpha}_{j}+\varepsilon_{i j}
$$

in which $\phi\left(\mathbf{z}_{i}^{\prime} \boldsymbol{\theta}\right)$ is some known function of a finite set of parameters $\boldsymbol{\theta} .^{16}$ This function is common across share equations and can be interpreted as the log of a general

\footnotetext{
${ }^{16}$ Blundell, Duncan and Pendakar (1998) compare the semiparametric specification used here with this more general alternative and find that it provides a good representation of demand behavior for households in the British FES used in this study.
} 
equivalence scale for household $i^{17}$. Interestingly, the extended partially linear model (3.10) is precisely the shape invariant specification considered in the work on pooling nonparametric regression curves by Härdle and Marron (1990) and Pinske and Robinson (1995).

To examine the shape invariant restrictions implicit in (3.10) we define $s=0,1, . ., S$ distinct household types of group size $n_{s}$ and let $\mathbf{z}^{s}$ represent the corresponding demographic structure for each group normalised such that for the base group $s=0$, $\phi\left(\mathbf{z}_{i}^{0 \prime} \boldsymbol{\theta}\right)=\mathbf{z}_{i}^{0 \prime} \boldsymbol{\alpha}_{j}=0$. The share equation for the base group (e.g. a couple with no children) becomes ${ }^{18}$

$$
w_{i j}^{0}=g_{j}^{0}\left(\ln x_{i}\right)+\varepsilon_{i j}^{0} .
$$

while for the remaining for $s=1, . ., S$ groups (e.g. couples with different numbers of children) the share equations become

$$
w_{i j}^{s}=g_{j}^{s}\left(\ln x_{i}-\phi\left(\mathbf{z}_{i}^{s \prime} \boldsymbol{\theta}\right)\right)+\mathbf{z}_{i}^{s \prime} \boldsymbol{\alpha}_{j}+\varepsilon_{i j}^{s}
$$

For any distinct household type $\mathbf{z}_{i}^{s}$ the shape invariance restrictions relative to the base group may be written

$$
g_{j}^{s}\left(\ln x_{i}\right)=g_{j}^{0}\left(\ln x_{i}-\phi\left(\mathbf{z}_{i}^{s \prime} \boldsymbol{\theta}\right)\right)+\mathbf{z}_{i}^{s \prime} \boldsymbol{\alpha}_{j}
$$

If the $\boldsymbol{\alpha}_{j}$ and $\boldsymbol{\theta}$ parameters for $j=1, \ldots . J-1$ were known then the shape restricted $g_{j}$ could be estimated by kernel regression on the transformed data $\ln x_{i}-\phi\left(\mathbf{z}^{s \prime} \boldsymbol{\theta}\right)$ and $w_{i j}^{s}-\mathbf{z}^{s \prime} \boldsymbol{\alpha}_{j}$, pooled across the household types $s=0,1, \ldots, S$. We replace the $\boldsymbol{\alpha}_{j}$ and $\boldsymbol{\theta}$ by $\sqrt{n}$ consistent estimators and note that the asymptotic properties of the kernel regression estimates of $g_{j}$ on the transformed data are unaffected. The choice of estimator for $\boldsymbol{\alpha}_{j}$ and $\boldsymbol{\theta}$ extends a method developed in the Härdle and Marron (1990)

\footnotetext{
${ }^{17}$ For example, we may choose $\phi\left(\mathbf{z}_{i}^{\prime} \boldsymbol{\theta}\right)=\ln \left(\mathbf{z}_{i}^{\prime} \boldsymbol{\theta}\right)$ where $\boldsymbol{\theta}$ is the vector of corresponding equivalence scales. See Pendakur (1998), for example.

${ }^{18}$ In the remainder of this subsection we suppress the bandwidth parameter and use superscripts to represent the different demographic groups.
} 
and Pinske and Robinson (1995) papers. The idea is to replace each $g_{j}^{s}\left(\ln x_{i}\right)$ by its unrestricted Nadaraya-Watson kernel regression estimator and choose $\boldsymbol{\alpha}_{j}$ and $\boldsymbol{\theta}$ so as to minimise some weighted quadratic loss. ${ }^{19}$

Define $\left(\widehat{\boldsymbol{\alpha}}_{j}, \widehat{\boldsymbol{\theta}}\right)$ as the value of $\left.\left(\boldsymbol{\alpha}_{j}, \boldsymbol{\theta}\right)\right)$ that minimises the integrated squared loss function

$$
L(\boldsymbol{\theta}, \boldsymbol{\alpha})=\sum_{s=1}^{S} \sum_{j=1}^{J-1} \int_{\underline{x}}^{\bar{x}}\left(\Lambda_{j s}\left(\ln x ; \boldsymbol{\theta}, \boldsymbol{\alpha}_{j}\right)\right)^{2} \pi_{s} \varpi_{j}(\ln x) d \ln x
$$

where $\boldsymbol{\alpha}^{\prime}=\left(\boldsymbol{\alpha}_{1}^{\prime}, \ldots, \boldsymbol{\alpha}_{J-1}^{\prime}\right)$ and where $\underline{x}$ and $\bar{x}$ are integration limits on the log of expenditure. The $\Lambda_{j s}$ term is given by

$$
\Lambda_{j s}\left(\ln x ; \boldsymbol{\theta}, \boldsymbol{\alpha}_{j}\right)=r_{j}^{s} f^{0}-f^{s} r_{j}^{0}\left(\ln x-\phi\left(\mathbf{z}^{s /} \widehat{\boldsymbol{\theta}}\right)\right)-\mathbf{z}^{s \prime} \boldsymbol{\alpha}_{j} f^{s} f^{0}\left(\ln x-\phi\left(\mathbf{z}^{s /} \widehat{\boldsymbol{\theta}}\right)\right)
$$

where $\pi_{s}$ is a group specific weight $\left(n_{s} / n\right.$ in our specification) and $\varpi_{j}(\ln x)$ is an equation-specific weighting function. ${ }^{20}$ This choice is equivalent to using $\left(f^{s} f^{0}(\ln x-\right.$ $\left.\phi\left(\mathbf{z}^{s /} \widehat{\boldsymbol{\theta}}\right)\right)^{2}$ as a weighting scheme for the Härdle and Marron (1990) estimator (3.14), and is precisely the estimator for random designs as suggested by Pinske and Robinson (1995). We apply this approach to our data using the predictions from the pooled model to estimate the $r_{j}^{s}$ terms in the loss function and implement a grid searching over a plausible range for $\phi$ to find the values for which the loss function attains a minimum within each year of our data. ${ }^{21}$

\footnotetext{
${ }^{19}$ In order to estimate these parameters there is no particular reason to use a kernel estimator for this shape invariant model. An attractive alternative semi-parametric estimator would be to adapt the sieve procedure in Ai and Chen (2000), for example.

${ }^{20}$ Note

$$
\begin{aligned}
g_{j}^{s}(\ln x)= & \mathbf{z}_{i}^{s \prime} \boldsymbol{\alpha}_{j}+g_{j}^{0}\left(\ln x-\phi\left(\mathbf{z}_{i}^{s \prime} \boldsymbol{\theta}\right)\right) \Longleftrightarrow \\
\widehat{f}^{0}\left(\ln x-\phi\left(\mathbf{z}_{i}^{s \prime} \boldsymbol{\theta}\right)\right) \widehat{r}_{j}^{s}(\ln x)= & \widehat{f}^{s}(\ln x) \widehat{r}_{j}^{0}\left(\ln x-\phi\left(\mathbf{z}_{i}^{s \prime} \theta\right)\right) \\
& +\widehat{f}^{s}(\ln x) \widehat{f}^{0}\left(\ln x-\phi\left(\mathbf{z}_{i}^{s \prime} \theta\right)\right) \mathbf{z}_{i}^{s \prime} \boldsymbol{\alpha}_{j}
\end{aligned}
$$

for all $x$. To eliminate the random denominators in the kernel regression terms $g_{j}^{s}$ and $g_{j}^{0}$, the expression (3.15) can be weighted by the product of densities $f^{s} f^{0}$ where $f^{s}$ is evaluated at $\ln x_{i}$ and $f^{0}$ at $\left(\ln x-\phi\left(\mathbf{z}^{\prime} \widehat{\boldsymbol{\theta}}\right)\right)$.

${ }^{21}$ We find that the optimum value for $\exp (\phi)$ lies in the range 1.2 to 1.5 over the period. We choose the value 1.29 which is very close to the average of our estimates, and is the OECD equivalence scale. See Blundell, Duncan and Pendakur (1988) for a further discussion of the estimation of this equivalence scale parameter.
} 
For the case where there are just two distinct groups $S=1$ and one equation $J-1=1$, Pinske and Robinson show $\sqrt{n}$-consistency and asymptotic normality of this estimator of $(\boldsymbol{\theta}, \boldsymbol{\alpha})$. They also show that the first order asymptotic properties of the kernel regression estimator of $\widehat{g}$ under the shape invariant restrictions are unaffected by the use of $\widehat{\boldsymbol{\alpha}}^{\prime}, \widehat{\boldsymbol{\theta}}$ in place of $\boldsymbol{\alpha}, \boldsymbol{\theta} .{ }^{22}$ As noted above the latter result is particularly useful in our case as we are not directly interested in $\boldsymbol{\alpha}^{\prime}, \boldsymbol{\theta}$ but rather in $g_{j}$. Proposition 5 below extends their conditions for $\sqrt{n}$-consistency of $\left(\widehat{\boldsymbol{\alpha}}^{\prime}, \widehat{\boldsymbol{\theta}}\right)$ to the more general case of many groups and many equations. Given this result we can then proceed to estimate the nonparametric Engel curves pooled across household types using the transformed variables $\left(w_{i j}-\mathbf{z}_{i}^{\prime} \widehat{\boldsymbol{\alpha}}_{j}\right)$ and $\left(\ln x_{i}-\phi\left(\mathbf{z}_{i}^{\prime} \widehat{\boldsymbol{\theta}}\right)\right) \cdot{ }^{23}$

Proposition 5 Let $\left(\widehat{\boldsymbol{\alpha}}^{\prime}, \widehat{\boldsymbol{\theta}}^{\prime}\right)$ be the values of $\left.\left(\boldsymbol{\alpha}_{j}, \boldsymbol{\theta}\right)\right)$ that minimise the integrated squared loss function (3.14). Under assumptions $A 1-A 8$ (see Appendix $A$ ), $\left(\widehat{\boldsymbol{\alpha}}^{\prime}, \widehat{\theta}^{\prime}\right)$ is a $\sqrt{n}$-consistent estimator for $\left(\boldsymbol{\alpha}_{0}^{\prime}, \theta_{0}^{\prime}\right)$.

Proof. See Appendix A.

One important requirement for this proposition to hold (Assumption A6 in Appendix A) is that $f^{s} f^{0}\left(\ln x-\phi\left(\mathbf{z}^{s \prime} \boldsymbol{\theta}\right)\right)$ is bounded away from zero at the true parameter value for $\boldsymbol{\theta}$. In our application we distinguish household types by family size with the base group being a couple without children and choose the log transformation for the equivalence scale function $\phi$. Since the scale for children relative to a childless couple is assumed to be bounded between zero and one half for each child, condition A6 is preserved.

\footnotetext{
${ }^{22}$ In proving this result Pinske and Robinson (1995) allow for a different bandwidth, $n h^{3} \longrightarrow \infty$ and $h \rightarrow 0$ as $n \rightarrow \infty$, which is more than satisfied by our choice of bandwidth which is proportional to $n^{\frac{1}{5}}$.

${ }^{23}$ An alternative estimator would be to adapt a minimum distance estimator for conditional moment restrictions. This could also allow for endogeneity using an intrumental variable approach. A first attempt at this for the shape invariant Engel curve model is presented in Blundell, Chen and Kristensen (2001).
} 


\subsection{Endogeneity of Total Expenditure}

To adjust for endogeneity we adapt the control function or augmented regression technique (see Holly and Sargan (1982), for example) to the semiparametric Engel curve framework. Consider first the nonparametric Engel curve (3.1). Suppose $\ln x$ is endogenous in the sense that for each commodity $j$

$$
E\left(\varepsilon_{i j} \mid \ln x_{i}\right) \neq 0 \text { or } E\left(w_{i j} \mid \ln x_{i}\right) \neq g_{j}\left(\ln x_{i}\right) .
$$

In this case the nonparametric estimator will not be consistent for the function of interest. To be precise, it will not provide the appropriate counterfactual: how will expenditure share patterns change for some ceteris paribus change in total expenditure?

Suppose there exist instrumental variables $\boldsymbol{\zeta}_{i}$ such that

$$
\ln x_{i}=\boldsymbol{\pi}^{\prime} \boldsymbol{\zeta}_{i}+v_{i} \text { with } E\left(v_{i} \mid \boldsymbol{\zeta}_{i}\right)=0
$$

In the application below we take the log of disposable income as the excluded instrumental variable for $\log$ total expenditure, $\ln x$. Further, we make the following key assumptions

$$
\begin{aligned}
E\left(w_{i j} \mid \ln x_{i}, \boldsymbol{\zeta}_{i}\right) & =E\left(w_{i j} \mid \ln x_{i}, v_{i}\right) \\
& =g_{j}\left(\ln x_{i}\right)+\rho_{j} v_{i} \forall j .
\end{aligned}
$$

This implies the augmented regression model

$$
w_{i j}=g_{j}\left(\ln x_{i}\right)+\rho_{j} v_{i}+\varepsilon_{i j} \forall j
$$

with

$$
E\left(\varepsilon_{i j} \mid \ln x_{i}\right)=0 \forall j
$$

Note that $g_{j}\left(\ln x_{i}\right)=E\left(w_{i j} \mid \ln x_{i}\right)-E\left(v_{i} \mid \ln x_{i}\right)$ eliminating $g_{j}\left(\ln x_{i}\right)$ using (3.21) yields

$$
w_{i j}-E\left(w_{i j} \mid \ln x_{i}\right)=\left(v_{i}-E\left(v_{i} \mid \ln x_{i}\right)\right) \rho_{j}+\varepsilon_{i j}
$$


which suggests a weighted instrumental variable estimator for $\rho_{j}$ by replacing the conditional means $E\left(w_{i j} \mid \ln x_{i}\right)$ and $E\left(v_{i} \mid \ln x_{i}\right)$ by their Nadaraya-Watson kernel regression estimators $\widehat{w}\left(\ln x_{i}\right)$ and $\widehat{v}\left(\ln x_{i}\right)$ respectively. Suitable instruments would be $I\left[\widehat{f}\left(\ln x_{i}\right)>b\right] . v_{i}$.

The resulting estimator of $g\left(\ln x_{i}\right)$ is given by

$$
\widehat{g}\left(\ln x_{i}\right)=\widehat{w}\left(\ln x_{i}\right)-\widehat{v}\left(\ln x_{i}\right) \widehat{\rho}_{j}
$$

Note that the unobservable error component $v$ in (3.23) is unknown. In estimation $v$ is replaced with the first stage reduced form residuals

$$
\widetilde{v}_{i}=\ln x_{i}-\widehat{\boldsymbol{\pi}}^{\prime} \boldsymbol{\zeta}_{i}
$$

where $\widehat{\boldsymbol{\pi}}$ is the least squares estimator of $\boldsymbol{\pi}$. Since $\widehat{\boldsymbol{\pi}}$ and $\widehat{\rho}$ converge at $\sqrt{n}$ the asymptotic distribution for $\widehat{g}\left(\ln x_{i}\right)$ follows the distribution of $\widehat{w}\left(\ln x_{i}\right)-\widehat{v}\left(\ln x_{i}\right) \rho_{j}$. Moreover, a test of the exogeneity null $H_{0}: \rho_{j}=0$, can be constructed from this least squares regression. ${ }^{24}$ In application we apply this procedure by augmenting the semiparametric model (3.10).

\subsection{Unobserved Heterogeneity}

We turn now to the relationship between nonparametric Engel curves and the average demands for a set of heterogeneous agents. For this discussion we omit dependence on observed characteristics $\mathbf{z}$. There are two alternative ways of interpreting the impact of heterogeneity on the average demands estimated from nonparametric Engel curve regression. We could assume individual demands are rational and then ask for conditions

\footnotetext{
${ }^{24}$ This method can be viewed as a special case of the method proposed in Newey, Powell and Vella (1999). They adopt a series estimator for the regression of $w$ on $\ln x$ and $v$. This generalises the form of (3.18) and (3.21). We chose not to follow the fully nonparametric control function approach here for two reasons. First in Blundell, Duncan and Pendakur (1998) it is shown that adding additional terms makes little difference for estimating Engel curves on a sample from a single year of British Family Expenditure Survey data. Second, for the computations in this study we would also have to make this adjustment for each share equation in each time period and also to adjust the asymptotics accordingly.
} 
on preferences and/or heterogeneity that imply rationality for average demands. This is the approach of McElroy (1987), Brown and Walker (1991) and Lewbel (1996). Alternatively, we could make no rationality assumptions on individual demands and simply ask what conditions enable average demands to satisfy rationality properties. This is the approach of Becker (1962), Grandmont (1992) and Hildenbrand (1994).

Suppose for each good $j$ we write average budget shares as

$$
E\left[w_{j} \mid \ln x, \mathbf{p}\right]=g_{j}(\ln x, \mathbf{p})
$$

then, if we let $\varepsilon$ represent a vector of unobserved heterogeneity terms with $E[\varepsilon \mid \ln \mathbf{x}, \mathbf{p}]=$ 0 , a necessary condition for the average budget shares recovered by the nonparametric analysis discussed above to be equal to average budget shares is that

$$
w_{j}=g_{j}(\ln x, \mathbf{p})+\phi_{j}(\ln x, \mathbf{p})^{\prime} \varepsilon .
$$

Notice this allows for quite different tastes across agents. In particular, the first-order price and income responses for agents can vary in any way. Thus a good may be a luxury for one person and a necessity for another.

The function $g_{j}(\ln x, \mathbf{p})$ gives mean responses to changes in prices conditional on a given level of total expenditure. Thus we can use this function for positive analysis, for example to recover the revenue implications from a change in taxes. Additionally, the utility function that is associated with an integrable set of demands $g_{j}(\ln x, \mathbf{p})$ is a prime candidate for use in equilibrium models that assume a representative agent. In our analysis below we apply the GARP tests to the mean function $g_{j}(\ln x, \mathbf{p})$. This averaging is very different to the standard aggregation structure in consumer theory developed by Gorman (1954) and Muellbauer (1976). In particular, we are not aggregating across different total budgets (incomes). Additionally, we are not assuming that individual demands are necessarily integrable; that is, for given $\varepsilon$ we can have that the Slutsky conditions may fail for $w_{j}(\ln x, \mathbf{p}, \varepsilon)$. In this respect, our structure is closer to that of 
Hildenbrand (1994) and Grandmont (1992). However, their analysis shows conditions for average demands to satisfy the Weak Axiom of Revealed Preference (WARP, see Varian (1982)) but GARP requires more. GARP implies the Slutsky symmetry conditions. If we wish to impose integrability at the individual level then there are restrictions on the $\phi_{j}(x, \mathbf{p})$ and the distribution of the heterogeneity terms (see McElroy (1987), Brown and Walker (1989) and Brown and Matzkin (1995)). ${ }^{25}$ Indeed, Brown and Walker (1989) show that for Slutsky symmetry to hold $\phi_{j}(x, \mathbf{p})$ must be either a function of $x$ or $\mathbf{p}$.

The reason that we are interested in testing for GARP using these mean responses is that without such a rationality condition holding, it is difficult to see how we would ever conduct coherent welfare analysis of non-marginal price changes. The heterogeneity conditions for using the mean function for the welfare analysis for consumers of a nonmarginal price change are, however, stronger than the conditions given in (3.27) which suffice for positive analysis. In an important paper McElroy (1987) considers the case of estimating cost function and share equation parameters for production analysis. For consumer welfare measures these results need to be extended. Consider the welfare measure based on the second-order approximation ${ }^{26}$ of the log cost function for a nonmarginal price change $\Delta \ln p_{j}$

$$
E\left[\frac{\Delta \ln c}{\Delta \ln p_{j}} \mid x, \mathbf{p}\right]=\left[w_{j} \mid x, \mathbf{p}\right]+\frac{1}{2} E\left[S_{j j} \mid x, \mathbf{p}\right] \Delta \ln p_{j} .
$$

where $S_{j j}$ is the Slutsky substitution term

$$
S_{j j}=\frac{\partial w_{j}}{\partial \ln p_{j}}+\frac{\partial w_{j}}{\partial \ln x} w_{j}
$$

Consequently in addition to the direct price effect on the share (3.28) includes the compensating income effect $\frac{\partial w_{j}}{\partial \ln x} w_{j}$. This introduces a bias term additional to that considered in McElroy (1987). Using (3.27) the mean welfare measure (3.28) has the form

\footnotetext{
${ }^{25}$ If all preference parameters are to be heterogeneous then preferences are essentially restricted to the class of Piglog demands (see Lewbel (1996), for example).

${ }^{26}$ See Banks, Blundell and Lewbel (1996), for example.
} 


$$
E\left[\frac{\Delta \ln c}{\Delta \ln p_{j}} \mid x, \mathbf{p}\right]=g_{j}+\frac{1}{2}\left(\frac{\partial g_{j}}{\partial \ln p_{j}}+\frac{\partial g_{j}}{\partial \ln x} g_{j}\right) \Delta \ln p_{j}+\frac{1}{2} \frac{\partial \phi_{j}^{\prime}}{\partial \ln x} \Omega_{\varepsilon} \phi_{j} \Delta \ln p_{j} .
$$

where $E\left\{\varepsilon \varepsilon^{\prime} \mid x, \mathbf{p}\right\}=\Omega_{\varepsilon}$. The first two terms on the right hand side of this expression can be computed using the mean function $g_{j}($.$) so that our mean function gives an exact$ first-order welfare effect. It also gives second order effects if the final bias term is zero. This will be the case if, for example, the heterogeneity term $\phi(\ln x, \mathbf{p})$ is independent of total expenditure so that all households have the same marginal income effects. ${ }^{27}$

In general the error term in (3.27) will represent measurement and optimisation error as well as preference heterogeneity so it would seem natural to work with local average demands. Averaging locally to each $x$ eliminates unobserved heterogeneity, measurement error and (zero mean) optimisation errors in demands but preserves any nonlinearities in the Engel curve relationship for each price regime.

\section{An Empirical Investigation on Repeated Cross-Sections}

\subsection{Data}

The data were drawn from the repeated cross-sections of household-level data in the British Family Expenditure Survey (1974 to 1993). The FES is a random sample of around 7,000 households per year. From this we used a sub-sample of all the two-adult households both those with and those without children ${ }^{28}$. The first and last percentiles of the within-year total expenditure distribution in this sub-sample was then trimmed out. This selection resulted in a sample size of 75,753 households (between 3,386 and 4,086 in each year). Expenditures on non-durable goods by these households were aggregated into 22 commodity groups and chained Laspeyres price indices for these

\footnotetext{
${ }^{27}$ Note, however, that this condition is sufficient and not necessary; weaker assumptions suffice to make the bias term zero or small.

${ }^{28} \mathrm{~A}$ further selection of households with cars was made in order to allow us to include motoring expenditures and, in particular, petrol as commodity groups.
} 
groups were calculated from the sub-indices of the UK Retail Price Index giving 20 annual price points for each of our 22 commodity groups.

The commodity groups are non-durable expenditures grouped into: beer, wine, spirits, tobacco, meat, dairy, vegetables, bread, other foods, food consumed outside the home, electricity, gas, adult clothing, children's clothing and footwear, household services, personal goods and services, leisure goods, entertainment, leisure services, fares, motoring and petrol ${ }^{29}$. Descriptive statistics for total nominal expenditure are given in Table D.1 of appendix D.

\subsection{Estimated Engel Curves and Normal Goods}

The nonparametric regression results are based on a Gaussian Nadaraya-Watson kernel estimation under the shape invariance restrictions (??). Adaptive kernel bandwidths ${ }^{30}$ were used throughout with the first round bandwidth chosen by cross-validation [cf. Härdle (1990)].

The three figures (4.1 to 4.3) below show the estimated Working-Leser Engel curves (budget share against log total nominal expenditure) for 3 of our 22 commodities, for 3 of our 20 periods (1975 (circles), 1980 (squares), 1985 (triangles)). These represent a typical necessity (bread), a luxury (entertainment) and beer which roughly displays a quadratic logarithmic Engel curve behaviour. On each Engel curve we plot the points on the chronological SMP paths which correspond to the 1st, 10th, 25th, 50th, 75th, 90th and 99th percentile points in the base year (1974). Pointwise 95\% confidence bands at these points are also drawn. Note that, as we would expect, the precision is much lower at the tails of the outlay distribution. The left to right drift of the Engel curves apparent in these figure illustrates the growth in nominal expenditure which took place

\footnotetext{
${ }^{29}$ More precise descriptions of components of the commodity groups are available from the authors.

${ }^{30}$ The adaptive bandwidth is $h=h \lambda$ where $h$ is the pilot bandwidth and $\lambda_{i}=\left[\frac{\widehat{f}^{h}\left(\ln x_{i}\right)}{\eta}\right]^{-\frac{1}{5}}$ where $\eta=\exp \left[n^{-1} \sum_{i} \ln \widehat{f}^{h}\left(\ln x_{i}\right)\right]$ see Blundell and Duncan (1998).
} 
Figure 4.1: The Engel curve for Bread

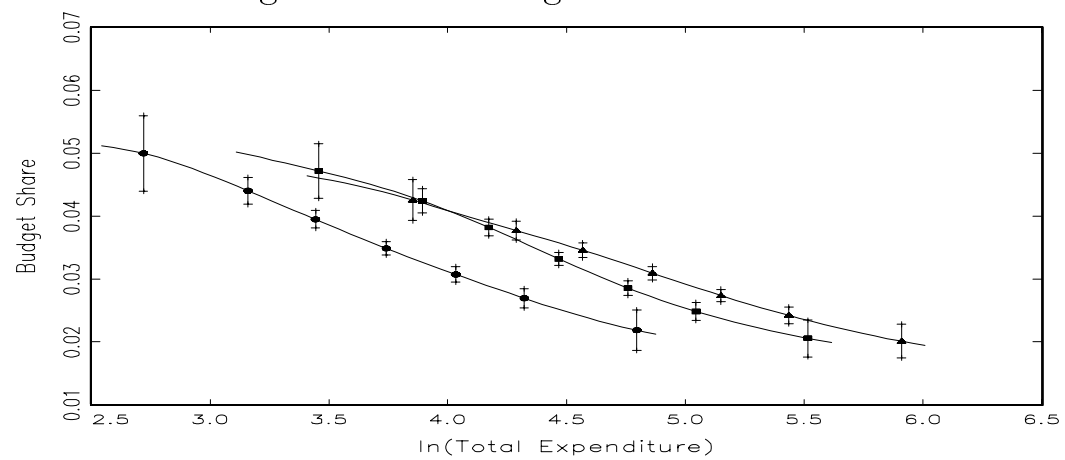

Figure 4.2: The Engel curve for Entertainment

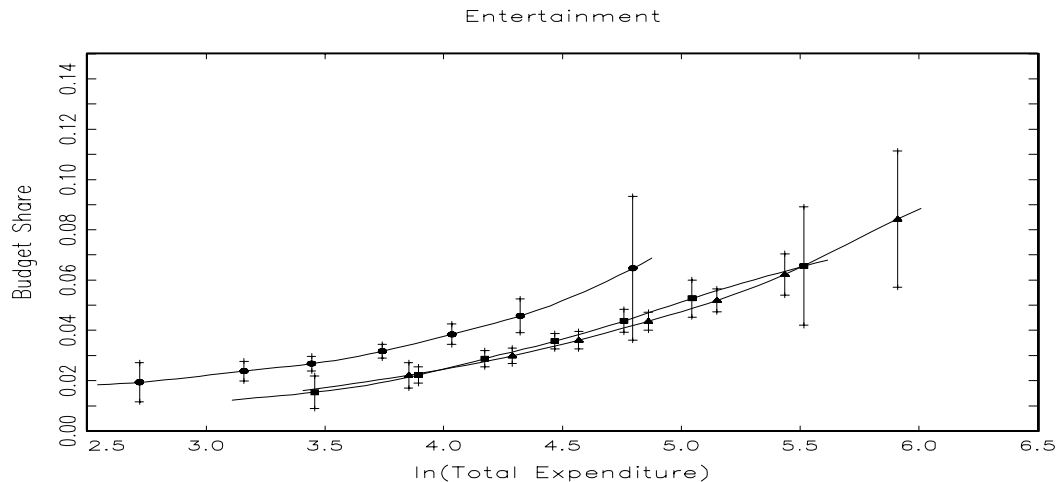

between these periods. ${ }^{31}$

\subsection{Testing GARP}

At each stage in the empirical analysis of the GARP conditions we will be comparing weighted sums of kernel regressions. The pairwise comparison $\mathbf{p}_{t}^{\prime} \mathbf{q}_{t}>\mathbf{p}_{t}^{\prime} \mathbf{q}_{s}$ can be written

\footnotetext{
${ }^{31} \mathrm{~A}$ full set of non-parametric regression results are available from the authors on request. These results confirm the normal goods assumption used in the discussion above.
} 
Figure 4.3: The Engel curve for Beer

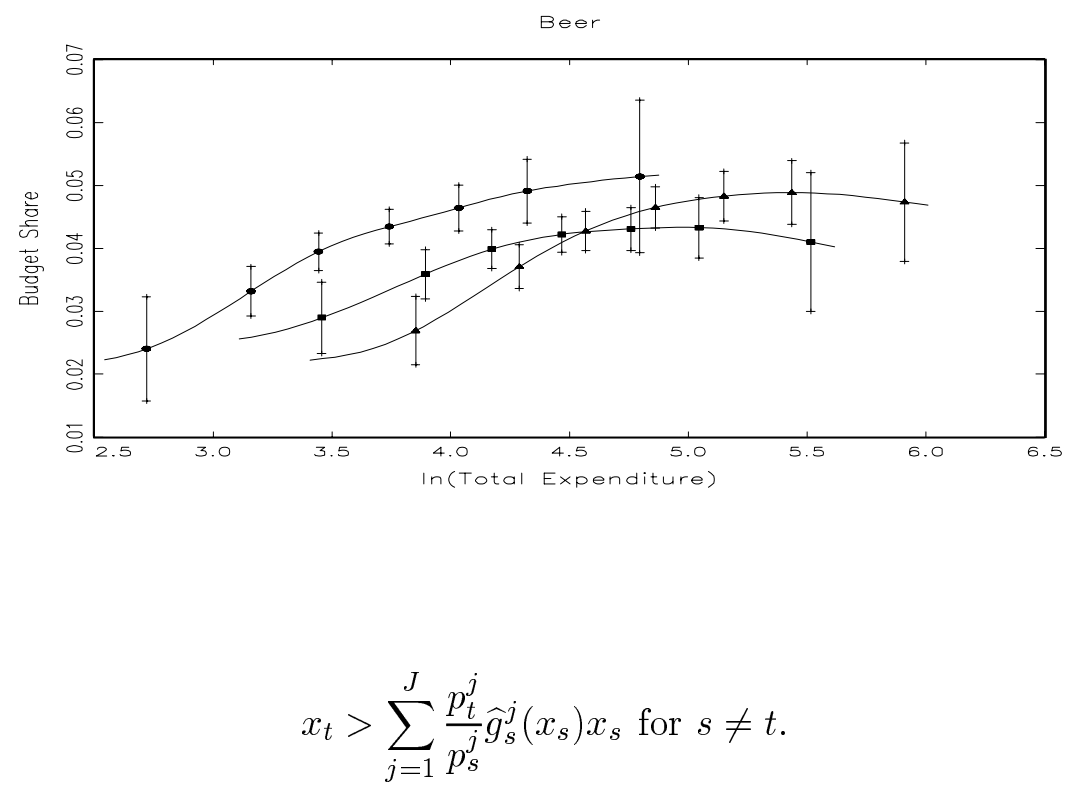

where $\widehat{g}_{s}^{j}\left(x_{s}\right)$ is the estimated budget share in equation (3.1). Noting that adding-up implies $\sum_{j=1}^{J} \widehat{g}_{t}^{j}\left(x_{t}\right) \equiv 1$ for all $t$, condition (4.1) conveniently reduces to the comparison

$$
\boldsymbol{\delta}_{t s}>\sum_{j=1}^{J-1} \gamma_{t s}^{j} \widehat{g}_{s}^{j}\left(x_{s}\right),
$$

where $\gamma_{t s}^{j}=\left(\frac{p_{t}^{j}}{p_{s}^{j}}-\frac{p_{t}^{J}}{p_{s}^{J}}\right)$ and $\delta_{t s}=\left(\frac{x_{t}}{x_{s}}-\frac{p_{t}^{J}}{p_{s}^{J}}\right)$ are known constant weights in each price regime.

To test GARP we will need to evaluate the inequality (4.2) at particular points on an SMP path. Since the nonparametric Engel curve has a pointwise asymptotic normal distribution we can evaluate the distribution of each $\widehat{g}_{t}^{j}(x)$ at any point $x .^{32}$ For (4.2) we need to find the distribution of the weighted sum of correlated kernel regression

\footnotetext{
${ }^{32}$ Briefly, for bandwidth choice $h$ and sample size $n$ the variance can be well approximated at point $x$ for large samples by$$
\operatorname{var}\left(g^{j}(x)\right) \simeq \frac{\sigma_{j}^{2}(x) c_{K}}{n h f_{h}(x)}
$$ 
estimates $\sum_{j=1}^{J-1} \gamma_{t s}^{j} \widehat{g}_{s}^{j}(x)$. However, since on any SMP path in any period the $\widehat{g}^{j}(x)$ kernel estimates for each good $j$ are to be evaluated using the same kernel smoother and the same bandwidth, the expression for the asymptotic variance of the weighted sum simplifies. In particular, the constants associated with the kernel function and the density $f_{h}(x)$ itself will be common to all variance and covariance terms. Pointwise standard errors and confidence bands for expression (4.2) are therefore tractable and are used extensively in the empirical application below.

When calculating demands on SMP paths we allow for the fact that the total expenditure levels in all periods except for the first are chosen on the basis of the estimated demands in the previous periods. For example, a SMP path constructed such that $\widetilde{x}_{t}=\mathbf{p}_{t}^{\prime} \mathbf{q}_{t}=\mathbf{p}_{t}^{\prime} \mathbf{q}_{t-1}\left(x_{t-1}\right)$, the expenditure level $\widetilde{x}_{t}$ is set such that $\widetilde{x}_{t}=$ $\sum_{j=1}^{J} \frac{p_{t}^{j}}{p_{t-1}^{j}} \widehat{g}_{t-1}^{j}\left(x_{t-1}\right) x_{t-1}$ and therefore $\widetilde{x}_{t}$ depends on the estimate of $\widehat{\mathbf{g}}_{t-1}\left(x_{t-1}\right)$ from the previous period. The test of $\mathbf{q}^{t-1} P^{0} \mathbf{q}^{t}$ requires that we have an estimate of $\operatorname{Var}\left(\delta_{t-1, t}-\gamma_{t-1, t}^{\prime} \widehat{\mathbf{g}}_{t}\right)$ and that this takes into account that $\widetilde{x}_{t}$ is set according to estimates of $\mathbf{g}_{t-1}\left(x_{t-1}\right)$ (and likewise that $\widetilde{x}_{s}$ is set according to estimates of $\widehat{\mathbf{g}}_{r}\left(\widetilde{x}_{r}\right)$ etc.). This is derived using the standard delta-method approach applied sequentially.

To implement our procedure we need to choose a set of SMP paths along which to evaluate GARP. To do this we select the starting points for each path to be at the 1st percentile, 1st decile, 1st quartile, median, 3rd quartile, 9th decile and 99th percentile points in the $x$ distribution for 1974 , the first year in our data set. The comparison where $c_{K}$ is a known constant and $f_{h}(x)$ is an (estimate) of the density of $x$

$$
\sigma_{j}^{2}(x)=n^{-1} \sum_{j=1}^{n} \omega_{j h}(x)\left(w_{i j}-g_{j}(x)\right)^{2}
$$

with weights from the kernel function

$$
\omega_{j h}(x)=K_{h}\left(x-x_{j}\right) / f_{h}(x)
$$

see Härdle (1990). 
Table 4.1: Number of rejections of GARP, by size of test.

\begin{tabular}{|l|c|c|c|c|c|}
\hline Starting point for & \multicolumn{5}{|c|}{$\alpha$} \\
\cline { 2 - 6 } each comparison path & $\mathbf{1 . 0}$ & $\mathbf{0 . 3 0}$ & $\mathbf{0 . 2 0}$ & $\mathbf{0 . 1 0}$ & $\mathbf{0 . 0 5}$ \\
\hline SMP paths: & & & & & \\
1st percentile & 1 & 0 & 0 & 0 & 0 \\
1st decile & 1 & 1 & 0 & 0 & 0 \\
1st quartile & 1 & 0 & 0 & 0 & 0 \\
Median & 1 & 1 & 1 & 1 & 0 \\
3rd quartile & 2 & 2 & 0 & 0 & 0 \\
9th decile & 11 & 6 & 3 & 1 & 0 \\
99th percentile & 28 & 21 & 1 & 0 & 0 \\
\hline Median path & 0 & 0 & 0 & 0 & 0 \\
Mean path & 0 & 0 & 0 & 0 & 0 \\
\hline
\end{tabular}

points for the following years are chosen along the SMP path as described in section 2.2. By Proposition 1 we know that if this path passes GARP then no path which preserves the same preference ordering will violate GARP. The annual median and mean (nonSMP) paths are also computed for comparison.

Table 4.1 shows the number and pattern of rejections for the system of 22 goods. Each column provides a count of the total number of rejections according to inequality (4.2). In each case a one sided test of size $\alpha$ is used, based on the pointwise asymptotic distribution of $\sum_{j=1}^{J-1} \gamma_{t s}^{j} g_{s}^{j}\left(x_{s}\right)$. The column headed $\alpha=1$ counts the number of rejects using inequality (4.2) directly without adjustment for estimation error in $g_{s}^{j}\left(x_{s}\right)$. In the remaining columns each inequality is adjusted by a one sided interval. From the first of these columns GARP can be seen to be rejected for a large number of points, especially in the upper tail of the outlay distribution. ${ }^{33}$ However, these rejections are not statistically significant. Very little adjustment is needed to dramatically reduce the

\footnotetext{
${ }^{33}$ Our interest is primarily in the points commonly used in the analysis of income distributions, i.e. interdecile points, interquartile points and the median. We include the 1st and the 99th percentile points for completeness.
} 
number of rejections. ${ }^{34}$

It is also interesting to observe that there are no rejections, even in the raw data, for the median or mean (non-SMP) paths. This is consistent with the observation which arises in tests of GARP on aggregate data that if the budget constraint is allowed to shift much either way between comparison points, as it does for median or mean total expenditure, then there is little chance of being able to find demands that cannot be rationalised.

\subsection{Continuous Sub-Periods Which Satisfy GARP}

Using the same set of SMP comparison points as in table 4.1, table 4.2 presents the continuous sub-periods of the data that satisfy GARP. For example, the chronological SMP path which starts at median total budget in 1974 runs into a violation of GARP when 1986 is added to the sequence. In this case it is the pair of years 1985 and 1986 which fail to satisfy GARP: the SMP path is constructed to reflect the ordering $\mathbf{q}_{86} R^{0} \mathbf{q}_{85}$ but we find that $\mathbf{q}_{85} P^{0} \mathbf{q}_{86}$, giving the violation.

Table 4.2: Continuous periods that satisfy GARP.

\begin{tabular}{|c|c|c|c|c|c|c|c|c|c|c|c|c|c|c|c|c|c|c|c|c|}
\hline & \multicolumn{20}{|c|}{ Periods } \\
\hline & 74 & 75 & 76 & 77 & 78 & 79 & 80 & 81 & 82 & 83 & 84 & 85 & 86 & 87 & 88 & 89 & 90 & \begin{tabular}{|l|}
91 \\
\end{tabular} & 92 & \begin{tabular}{|l|}
93 \\
\end{tabular} \\
\hline $1 \mathrm{st}$ & & & & & & & & & & & & & & & & & & & & \\
\hline 10th & & & & & & & & & & & & & & & & & & & & \\
\hline 25 th & & & & & & & & & & & & & & & & & & & & \\
\hline 50 th & & & & & & & & & & & & & & & & & & & & \\
\hline 75 th & & & & & & & & & & & & & & & & & & & & \\
\hline 90th & & & & & & & & & & & & & & & & & & & & \\
\hline 99th & & & & & & & & & & & & & & & & & & & & \\
\hline
\end{tabular}

\footnotetext{
${ }^{34}$ We have not attempted to compute the size of the implicit joint test.
} 
Interestingly, the table also shows the largest continuous sub-period in which we are able to bound the indifference curve. For example, using the reference demand bundle at median total outlay in 1974 we are able to bound a curve using the expansion paths and price data for 1974 to 1985 inclusive (we are also able to bound curves using reference demands at any within-year median total expenditure level or reference demands at any point on the on the chronological SMP path between 1974 and 1985). However, if we add 1986 to the set of admissible periods the algorithm fails to converge. We then start again using the 1986 point on the median SMP path as our starting point. In all, for the median we find the entire period separates down into two sub-periods within which we are able to bound an indifference curve. Similarly the 1st and 9th decile paths break into two and four sub-periods respectively, while the 99 th percentile breaks down into five.

We can use this knowledge of periods in which GARP is satisfied in a number of ways. To illustrate two of them we present bounds on the base-period reference cost-of-living index, and bounds on year-to-year inflation rates.

\subsection{Bounds on the True Cost-of-living Index}

Table 4.2 shows that preferences on the SMP paths starting at the 10th and 90th percentile points, the quartiles and the median of the base period total budget distribution all satisfy integrability at least up until 1985. We use the data for this period and the algorithms described in section 2.4 to bound the true cost-of-living index $c\left(\mathbf{p}_{85}, \mathbf{q}_{74}\right) / c\left(\mathbf{p}_{75}, \mathbf{q}_{74}\right)$ for a reference demand bundle at each of these points in the 1974 total budget distribution. Figure 4.4 shows the bounds for each reference budget in 1985 , with $1974=1000$. It is interesting to note that the bounds for 10 th and 90 th percentile points do not overlap and indicate greater rise on the cost of living for poorer, compared to richer, households over this period.

We also compare the performance of the GARP bounds for the true index with other 
Figure 4.4: GARP Cost of living index bounds 1985 by percentile point (1974=1000).

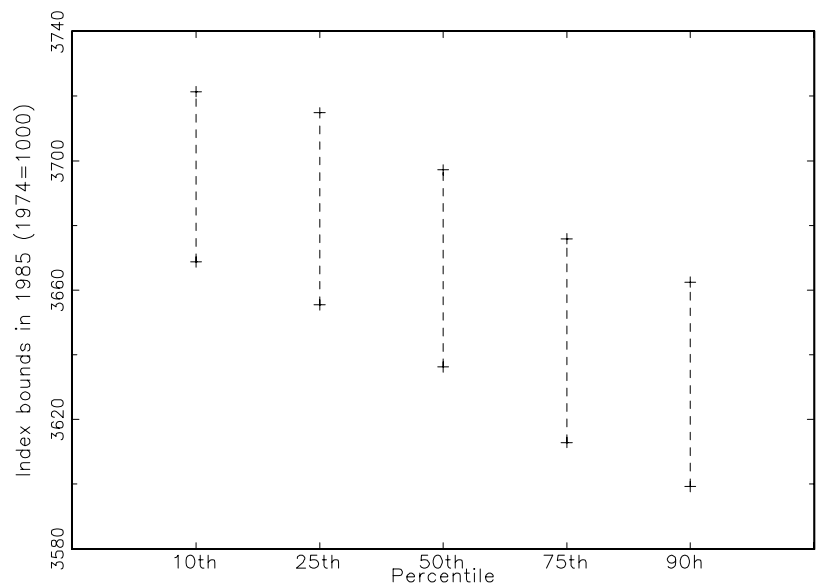

nonparametric bounds and other popular price index formulae over a longer period. This is shown in table 4.3. The first panel shows the price index numbers for the Paasche, Laspeyres and the chained Törnqvist. These indices can also be thought of as corresponding exactly to true indices under various assumptions regarding the precise form of preferences ${ }^{35}$. The second panel in table 4.3 shows various nonparametric bounds on the true index referenced at $\mathbf{q}_{74}$ where $\mathbf{q}_{74}=\mathbf{q}_{74}(x)$ evaluated at 1974 median total budget. The bounds provided by Lerner (1935-36) are simply a reflection of the idea that the true index (being a weighted average of price changes) must lie somewhere between the maximum and the minimum ratio of the price changes of all goods: i.e.

$$
\min _{j}\left\{\frac{p_{t}^{j}}{p_{74}^{j}}: j=1, \ldots, J\right\} \leq \frac{c\left(\mathbf{p}_{t}, \mathbf{q}_{74}\right)}{c\left(\mathbf{p}_{74}, \mathbf{q}_{74}\right)} \leq \max _{j}\left\{\frac{p_{t}^{j}}{p_{74}^{j}}: j=1, \ldots, J\right\} .
$$

Pollak (1971) improves this by linking Lerner's result with the original Konüs (1924) result that the Laspeyres index approximates the true base-referenced cost of living

\footnotetext{
${ }^{35}$ The Paasche and Laspeyres, for example, are exact for Leontief preferences, the Törnqvist is exact for translog.
} 
index from above, i.e.

$$
\min _{j}\left\{\frac{p_{t}^{j}}{p_{74}^{j}}: j=1, \ldots, J\right\} \leq \frac{c\left(\mathbf{p}_{t}, \mathbf{q}_{74}\right)}{c\left(\mathbf{p}_{74}, \mathbf{q}_{74}\right)} \leq \frac{\mathbf{p}_{t}^{\prime} \mathbf{q}_{74}}{\mathbf{p}_{74}^{\prime} \mathbf{q}_{74}} .
$$

The bounds from classical revealed preference restrictions of the type used by Varian (1982) and calculated using the demands in each period at median within-period total budget are also reported (labelled classical RP). None of these nonparametric solutions have any trouble in providing bounds for the entire period. The classical bounds for example, do not violate GARP for the reasons explained above. However, the bounds derived by our method must take account of the break between 1985 and 1986. This is because when we seek to derive the bounds using the data from both 1985 and 1986 the algorithms do not converge (convergence requires GARP as shown in propositions 2 and 3). Instead we bound the indifference curves using prices and expansion paths from all periods excluding 1986. We then use these to bound the cost-of-living index using all of the price data (including 1986) as described in 2.2 .

We find, confirming the results in Varian (1982) and Manser and McDonald (1988), that classical non-parametric/revealed preference bounds based on the median demand data gives little additional information on the curvature of the indifference curve through commodity space and hence the bounds on the true index are wide. However, by the use of expansion paths we can dramatically improve these bounds. This is illustrate in figure 4.5 in which the GARP bounds are represented by the solid lines and the classical $\mathrm{RP}$ bounds by the dashed line.

Comparing the GARP bounds on the true, fixed base cost of living index to the three price index number formulae we see that the chained Törnqvist. performs the best as an empirical approximation to the true index ${ }^{36}$. This is despite the fact that,

\footnotetext{
${ }^{36}$ Comparisons with other price indices are available from the authors.
} 
Table 4.3: Popular price indices, nonparametric and GARP bounds, 1974 to 1993.

\begin{tabular}{|c|c|c|c|c|c|c|c|}
\hline \multirow[b]{2}{*}{ Year } & \multicolumn{3}{|c|}{ Price Indices } & \multicolumn{4}{|c|}{ "Nonparametric/RP bounds } \\
\hline & $\mathrm{P}$ & $\mathrm{L}$ & $\mathrm{T}$ & Lerner & Pollak & Classical RP & GARP \\
\hline 74 & 1000 & 1000 & 1000 & 1000 & 1000 & 1000 & 1000 \\
\hline 75 & 1215 & 1232 & 1223 & {$[1025,1721]$} & {$[1025,1232]$} & {$[1206,1232]$} & {$[1214,1228]$} \\
\hline 76 & 1516 & 1530 & 1528 & {$[1182,1985]$} & {$[1182,1530]$} & {$[1431,1530]$} & {$[1514,1530]$} \\
\hline 77 & 1762 & 1787 & 1783 & {$[1239,2590]$} & {$[1239,1787]$} & {$[1700,1787]$} & {$[1761,1781]$} \\
\hline 78 & 1931 & 1957 & 1960 & {$[1385,2513]$} & ][1385,1957] & {$[1894,1957$} & {$[1936,1957]$} \\
\hline 79 & 2086 & 2119 & 2121 & {$[1461,2636]$} & {$[1461,2119]$} & {$[2058,2119]$} & {$[2093,2119]$} \\
\hline 80 & 2463 & 2514 & 2514 & {$[1734,3142]$} & {$[1734,2514]$} & {$[2442,2514]$} & {$[2478,2509]$} \\
\hline 81 & 2780 & 2841 & 2841 & {$[1770,4077]$} & {$[1770,2841]$} & {$[2687,2841]$} & {$[2801,2838]$} \\
\hline 82 & 3093 & 3189 & 3178 & {$[1821,4287]$} & {$[1821,3189]$} & {$[2983,3189]$} & {$[3123,3172]$} \\
\hline 83 & 3260 & 3381 & 3371 & {$[1828,4924]$} & {$[1828,3381]$} & {$[3197,3381]$} & {$[3314,3369]$} \\
\hline 84 & 3408 & 3558 & 3534 & {$[1790,4921]$} & {$[1790,3558]$} & {$[3335,3558]$} & {$[3473,3530]$} \\
\hline 85 & 3551 & 3733 & 3700 & {$[1836,5086]$} & {$[1836,3733]$} & {$[3546,3733]$} & {$[3634,3696]$} \\
\hline 86 & 3700 & 3911 & 3876 & {$[1900,5463]$} & {$[1900,3911]$} & {$[3595,3911]$} & {$[3808,3873]$} \\
\hline 87 & 3825 & 4035 & 3991 & {$[1920,6049]$} & {$[1920,4035]$} & {$[3626,4035]$} & {$[3918,3989]$} \\
\hline 88 & 3922 & 4163 & 4113 & {$[1923,6143]$} & {$[1923,4163]$} & {$[3702,4163]$} & {$[4036,4110]$} \\
\hline 89 & 4130 & 4379 & 4322 & {$[1996,6397]$} & {$[1996,4379]$} & {$[3688,4379]$} & {$[4240,4319]$} \\
\hline 90 & 4406 & 4669 & 4608 & {$[2079,6637]$} & {$[2079,4669]$} & {$[3739,4669]$} & {$[4521,4604]$} \\
\hline 91 & 4723 & 5044 & 4967 & {$[2109,7507]$} & {$[2109,5044]$} & {$[4073,5044]$} & {$[4871,4963]$} \\
\hline 92 & 4996 & 5437 & 5323 & {$[2091,8353]$} & {$[2091,5437]$} & {$[4038,5437]$} & {$[5214,5318]$} \\
\hline 93 & 5177 & 5650 & 5499 & {$[2066,9098]$} & {$[2066,5650]$} & {$[3990,5650]$} & {$[5382,5493]$} \\
\hline
\end{tabular}

Notes: $\mathrm{P}=$ Paasche. $\mathrm{L}=$ Laspeyres, $\mathrm{T}=$ Chained Törnqvist/Divisa

as an index in which reference utility is updated in each period, the Törnqvist. cannot strictly be compared to a fixed base true index. The Laspeyres, which is a first order approximation to the true index in question, understates the true increase in the cost of living by between about $3 \%$ and $5 \%$ by the end of the period.

\subsection{Bounds on Annual Inflation Rates}

As well as deriving bounds on fixed base cost-of-living indices, revealed preference restrictions can also be calculated on annual inflation rates in which the reference demand bundle is updated in each period. The 1990 annual inflation bound 
Figure 4.5: GARP bounds and classical RP bounds, 1974 to 1993.

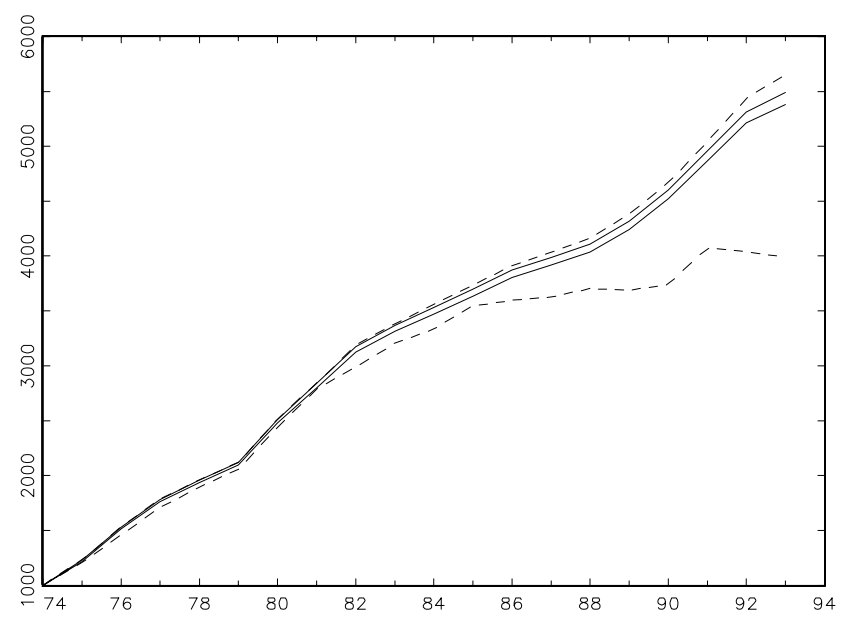

rate for example is calculated from the bound on the 1989-based cost-of-living index $c\left(\mathbf{p}_{90}, \mathbf{q}_{89}\right) / c\left(\mathbf{p}_{89}, \mathbf{q}_{89}\right)$. The results are shown in Table 4.4 where the improvement afforded by the GARP bounds over the previously available nonparametric bounds is apparent. Indeed the tightness of the GARP bounds is remarkable. Again the Törnqvist performs the best of the index number formulae followed by the Laspeyres which is often close to the top of the GARP bounds. Note that the inflation rate for the year to 1986 is missing from the GARP bounds because of the GARP violation between these two years.

\section{Summary and Conclusions}

In this paper we have applied nonparametric statistical methods to the nonparametric theory of consumer demand. We exploit the idea that price taking individuals in the same market at the same time face the same relative prices, in order to smooth across the demands of individuals for each common price regime. We first show that knowledge of budget expansion paths can improve the power of nonparametric tests of 
Table 4.4: Annual inflation rates for popular price indices, nonparametric and GARP bounds, 1975 to 1993 .

\begin{tabular}{c|ccc|cccc}
\hline \hline \multirow{2}{*}{ Year } & \multicolumn{3}{|c|}{ Price Indices } & \multicolumn{4}{|c}{ Nonparametric/RP bounds } \\
\cline { 2 - 8 } & $\mathrm{P}$ & $\mathrm{L}$ & $\mathrm{T}$ & Lerner & Pollak & Classical RP & GARP \\
\hline 75 & 21.48 & 23.16 & 22.28 & {$[2.50,72.10]$} & {$[2.50,23.16]$} & {$[20.59,23.16]$} & {$[\mathbf{2 1 . 4 7 , 2 2 . 8 5}]$} \\
76 & 24.60 & 25.27 & 24.93 & {$[5.00,54.13]$} & {$[5.00,25.27]$} & {$[17.86,25.27]$} & {$[\mathbf{2 4 . 5 7 , 2 5 . 2 7}]$} \\
77 & 16.54 & 16.89 & 16.71 & {$[4.82,30.51]$} & {$[4.82,16.89]$} & {$[13.82,16.89]$} & {$[\mathbf{1 6 . 5 0 , 1 6 . 8 0}]$} \\
78 & 9.85 & 10.05 & 9.95 & {$[-12.93,20.17]$} & {$[-12.93,10.05]$} & {$[-12.93,10.05]$} & {$[\mathbf{9 . 8 3 , 1 0 . 0 5}]$} \\
79 & 8.13 & 8.31 & 8.22 & {$[0.17,15.39]$} & {$[0.17,8.31]$} & {$[0.17,8.31]$} & {$[\mathbf{8 . 1 3 , 8 . 3 1}]$} \\
80 & 18.29 & 18.74 & 18.51 & {$[7.75,49.92]$} & {$[7.75,18.74]$} & {$[8.00,18.74]$} & {$[\mathbf{1 8 . 5 6 , 1 8 . 5 5}]$} \\
81 & 12.92 & 13.11 & 13.02 & {$[2.08,29.76]$} & {$[2.08,13.11]$} & {$[9.22,13.11]$} & {$[\mathbf{1 3 . 1 1 , 1 3 . 1 1}]$} \\
82 & 11.53 & 12.18 & 11.85 & {$[-0.93,32.21]$} & {$[-0.93,12.18]$} & {$[8.69,12.18]$} & {$[\mathbf{1 1 . 5 1 , 1 2 . 0 2}]$} \\
83 & 5.95 & 6.18 & 6.06 & {$[-6.97,25.32]$} & {$[-6.97,6.18]$} & {$[-6.97,6.18]$} & {$[\mathbf{6 . 0 5 , 6 . 1 4}]$} \\
84 & 4.80 & 4.91 & 4.86 & {$[-2.06,21.00]$} & {$[-2.06,4.91]$} & {$[-2.06,4.91]$} & {$[\mathbf{4 . 8 8 , 4 . 9 1}]$} \\
85 & 4.65 & 4.72 & 4.69 & {$[-3.18,12.77]$} & {$[-3.18,4.72]$} & {$[-3.18,4.72]$} & {$[\mathbf{4 . 7 2 , 4 . 7 2}]$} \\
86 & 4.77 & 4.73 & 4.75 & {$[0.70,8.64]$} & {$[0.70,4.73]$} & {$[1.36,4.73]$} & \\
87 & 2.89 & 3.04 & 2.97 & {$[-10.30,10.73]$} & {$[-10.30,3.04]$} & {$[-0.22,3.04]$} & {$[\mathbf{2 . 8 8 , 3 . 0 4}]$} \\
88 & 3.06 & 3.04 & 3.05 & {$[-4.50,8.87]$} & {$[-4.50,3.04]$} & {$[-0.60,3.04]$} & {$[\mathbf{3 . 0 4 , 3 . 0 4}]$} \\
89 & 5.07 & 5.11 & 5.09 & {$[-0.39,10.48]$} & {$[-0.39,5.11]$} & {$[-0.39,5.11]$} & {$[\mathbf{5 . 0 6 , 5 . 1 0}]$} \\
90 & 6.59 & 6.65 & 6.62 & {$[1.38,10.50]$} & {$[1.38,6.65]$} & {$[1.38,6.65]$} & {$[\mathbf{6 . 6 3 , 6 . 6 3}]$} \\
91 & 7.78 & 7.81 & 7.80 & {$[1.45,14.94]$} & {$[1.45,7.81]$} & {$[8.04,7.81]$} & {$[\mathbf{7 . 8 1 , 7 . 8 1}]$} \\
92 & 7.07 & 7.24 & 7.16 & {$[-0.85,18.90]$} & {$[-0.85,7.24]$} & {$[6.59,7.24]$} & {$[\mathbf{7 . 0 9 , 7 . 2 4}]$} \\
93 & 3.28 & 3.34 & 3.31 & {$[-8.03,9.58]$} & {$[-8.03,3.34]$} & {$[-7.69,3.34]$} & {$[\mathbf{3 . 3 4 , 3 . 3 4}]$} \\
\hline \hline
\end{tabular}

Notes: $\mathrm{P}=$ Paasche. $\mathrm{L}=$ Laspeyres, $\mathrm{T}=$ Törnqvist/Divisa

revealed preference theory. In cases in which revealed preference conditions are violated we could use an Afriat-Varian conditional demand approach but we leave that for future work. We also show how budget expansion paths can be used to place tight bounds on level sets of utility in commodity space and hence to provide tight nonparametric bounds on true cost-of-living indices. We present algorithms for the computation of these bounds.

Expansion paths can be estimated by nonparametric Engel curves and this is shown to provide a useful stochastic structure within which to examine the consistency of individual data and revealed preference theory. The implications for pooling across 
households with different demographic composition are also examined and an appropriate semiparametric estimator is derived.

Using a long time series of repeated cross-sections from the 1974-1993 British Family Expenditure Surveys we estimate semiparametric Engel curves and examine whether revealed preference theory is rejected. We show that GARP is not rejected for long periods, particularly when we allow for sampling/stochastic variation. We derive bounds on cost-of-living indices from our analysis which are much tighter than those based on the revealed preference restrictions implied by demands at, say, annual mean total expenditure. We also note that the chained Törnqvist (approximate Divisia) cost-of-living index performs well as a empirical approximation to the true base-period referenced index. 


\section{Appendices}

\section{A. Proofs of Lemmas and Propositions}

\section{Proof of Proposition 1}

Without loss of generality we take the GARP rejecting preference ordered sub-sequence to be $\left\{\mathbf{q}_{s}\left(\hat{x}_{s}\right), \mathbf{q}_{t}\left(\hat{x}_{t}\right), \mathbf{q}_{u}\left(\hat{x}_{u}\right)\right\}$. We have:

(1) $\hat{x}_{s}=\mathbf{p}_{s}^{\prime} \mathbf{q}_{s}\left(\hat{x}_{s}\right) \geq \mathbf{p}_{s}^{\prime} \mathbf{q}_{t}\left(\hat{x}_{t}\right)$ and

(2) $\hat{x}_{t}=\mathbf{p}_{t}^{\prime} \mathbf{q}_{t}\left(\hat{x}_{t}\right) \geq \mathbf{p}_{t}^{\prime} \mathbf{q}_{u}\left(\hat{x}_{u}\right)$ and

(3) $\hat{x}_{u}=\mathbf{p}_{u}^{\prime} \mathbf{q}_{u}\left(\hat{x}_{u}\right)>\mathbf{p}_{u}^{\prime} \mathbf{q}_{s}\left(\hat{x}_{s}\right)$.

We consider the SMP path for this preference ordered sub-sequence and show that it too rejects GARP. The SMP path $\left\{\mathbf{q}_{s}\left(\tilde{x}_{s}\right), \mathbf{q}_{t}\left(\tilde{x}_{t}\right), \mathbf{q}_{u}\left(\hat{x}_{u}\right)\right\}$ has:

(4) $\tilde{x}_{t}=\mathbf{p}_{t}^{\prime} \mathbf{q}_{t}\left(\tilde{x}_{t}\right)=\mathbf{p}_{t}^{\prime} \mathbf{q}_{u}\left(\hat{x}_{u}\right)$ and

(5) $\tilde{x}_{s}=\mathbf{p}_{s}^{\prime} \mathbf{q}_{s}\left(\tilde{x}_{s}\right)=\mathbf{p}_{s}^{\prime} \mathbf{q}_{t}\left(\tilde{x}_{t}\right)$.

By construction this is a preference ordered sub-sequence $\left(\mathbf{q}_{t}\left(\tilde{x}_{t}\right) R^{0} \mathbf{q}_{u}\left(\hat{x}_{u}\right)\right.$ and $\left.\mathbf{q}_{s}\left(\tilde{x}_{s}\right) R^{0} \mathbf{q}_{t}\left(\tilde{x}_{t}\right)\right)$

so that this sub-sequence rejects GARP if $\mathbf{q}_{u}\left(\hat{x}_{u}\right) P^{0} \mathbf{q}_{s}\left(\tilde{x}_{s}\right)$; that is, if:

(6) $\mathbf{p}_{u}^{\prime} \mathbf{q}_{u}\left(\hat{x}_{u}\right)>\mathbf{p}_{u}^{\prime} \mathbf{q}_{s}\left(\tilde{x}_{s}\right)$.

Conditions (2) and (4) imply $\mathbf{p}_{t}^{\prime} \mathbf{q}_{t}\left(\hat{x}_{t}\right) \geq \mathbf{p}_{t}^{\prime} \mathbf{q}_{t}\left(\tilde{x}_{t}\right)$ which implies $\hat{x}_{t} \geq \tilde{x}_{t}$.

This and conditions (1) and (5) give:

$$
\mathbf{p}_{s}^{\prime} \mathbf{q}_{s}\left(\hat{x}_{s}\right) \geq \mathbf{p}_{s}^{\prime} \mathbf{q}_{t}\left(\hat{x}_{t}\right) \geq \mathbf{p}_{s}^{\prime} \mathbf{q}_{t}\left(\tilde{x}_{t}\right)=\mathbf{p}_{s}^{\prime} \mathbf{q}_{s}\left(\tilde{x}_{s}\right)
$$

which implies $\hat{x}_{s} \geq \tilde{x}_{s}$. Finally, condition (3) and normality imply $\mathbf{p}_{u}^{\prime} \mathbf{q}_{u}\left(\hat{x}_{u}\right)>\mathbf{p}_{u}^{\prime} \mathbf{q}_{s}\left(\hat{x}_{s}\right) \geq$ $\mathbf{p}_{u}^{\prime} \mathbf{q}_{s}\left(\tilde{x}_{s}\right)$ which is condition (6); hence GARP is rejected for this sub-sequence.

\section{Proof of Proposition 2.}

PART A. We denote the sets $F$ and $W$ at the end of iteration $s$ by $F^{(s)}$ and $W^{(s)}$ respectively, with elements $\left\{\mathbf{q}_{1}, \mathbf{q}_{2}^{(s)}, \ldots \mathbf{q}_{T}^{(s)}\right\}$.

The first step is to show that as we iterate we never move 'up' an expansion path. Formally, at iteration $s+1$ we have:

$$
\mathbf{q}_{t}^{(s+1)} \leq \mathbf{q}_{t}^{(s)}
$$

To see this, consider $\mathbf{q}_{t}^{(s+1)} \in F^{(s+1)}$. At iteration $s+1$ step (2) of the algorithm takes the min over all $\mathbf{q}_{w} \in W^{(s)}$ so that $\mathbf{p}_{t}^{\prime} \mathbf{q}_{t}^{(s+1)} \leq \mathbf{p}_{t}^{\prime} \mathbf{q}_{w}$ for all $\mathbf{q}_{w} \in W^{(s)}=F^{(s)}$. Since the latter contains $\mathbf{q}_{t}^{(s)}$ we have the claimed inequality.

The next step is to show that if GARP and weak normality hold then for any pair $(t, u)$ and $k \geq 0$ we have:

$$
\mathbf{q}_{t}^{(s+1)}=\mathbf{q}_{t}\left(\mathbf{p}_{t}^{\prime} \mathbf{q}_{u}^{(s)}\right)<\mathbf{q}_{t}^{(s)} \Rightarrow \mathbf{q}_{u}^{(s)}<\mathbf{q}_{u}\left(\mathbf{p}_{u}^{\prime} \mathbf{q}_{t}^{(s-k)}\right)
$$


This is a 'no swapping' condition which states that if we change $\mathbf{q}_{t}$ to be just revealed preferred to $\mathbf{q}_{u}^{(s)}$ then we never have that $\mathbf{q}_{u}^{(s)}$ is revealed preferred to $\mathbf{q}_{t}^{(s)}$. To show this, note that the equality on the left hand side of the implication implies that $\mathbf{q}_{t}^{(s+1)} R \mathbf{q}_{u}^{(s)}$ . GARP and the inequality on the lhs of the implication give:

$$
\mathbf{p}_{u}^{\prime} \mathbf{q}_{u}^{(s)} \leq \mathbf{p}_{u}^{\prime} \mathbf{q}_{t}^{(s+1)}<\mathbf{p}_{u}^{\prime} \mathbf{q}_{t}^{(s)}
$$

With the monotonicity result above and noting that $k \geq 0$ this implies:

$$
\mathbf{q}_{u}^{(s)}=\mathbf{q}_{u}\left(\mathbf{p}_{u}^{\prime} \mathbf{q}_{u}^{(s)}\right)<\mathbf{q}_{u}\left(\mathbf{p}_{u}^{\prime} \mathbf{q}_{t}^{(s)}\right) \leq \mathbf{q}_{u}\left(\mathbf{p}_{u}^{\prime} \mathbf{q}_{t}^{(s-k)}\right)
$$

as claimed.

Now define the set:

$$
\bar{Q}\left(\mathbf{q}_{1}\right)=\left\{\mathbf{q}: \mathbf{q} \text { is on some SMP path that finishes at } \mathbf{q}_{1}\right\}
$$

Thus we take all the sub-sequences of $\{2,3 \ldots T\}$ (for example, $\{4,2,7\}$ ), construct the SMP paths that finish at $\mathbf{q}_{1}$ and include all the points on these paths in $\bar{Q}\left(\mathbf{q}_{1}\right)$. Since there are only a finite number of permutations of subsets of $\{2,3 \ldots T\}$ this is a finite set. We now show that if a demand ever enters $F$ then it is in this set; formally:

$$
\mathbf{q}_{t}^{(s)} \in \bar{Q}\left(\mathbf{q}_{1}\right)
$$

We prove this by induction. Consider first $F^{(1)}$ and $\mathbf{q}_{t}^{(1)}$. Step 2 of the algorithm gives for some $u$ we have $\mathbf{q}_{t}^{(1)}=\mathbf{q}_{t}\left(\mathbf{p}_{t}^{\prime} \mathbf{q}_{u}\left(\mathbf{p}_{u}^{\prime} \mathbf{q}_{1}\right)\right)$. Thus $\left\{\mathbf{q}_{t}^{(1)}, \mathbf{q}_{u}\left(\mathbf{p}_{u}^{\prime} \mathbf{q}_{1}\right), \mathbf{q}_{1}\right\}$ is an SMP path (with no second element if $u=t$ ). Thus all the elements of $F^{(1)}$ are contained in $\bar{Q}\left(\mathbf{q}_{1}\right)$. To continue with the induction proof, suppose that all of the elements of $F^{(s)}$ are on SMP paths. To show that all of the elements of $F^{(s+1)}$ are on SMP paths, we need only consider an element that changes between iterations $s$ and $s+1$ :

$$
\mathbf{q}_{t}^{(s+1)}=\mathbf{q}_{t}\left(\mathbf{p}_{t}^{\prime} \mathbf{q}_{u}^{(s)}\right)<\mathbf{q}_{t}^{(s)}
$$

Since $\mathbf{q}_{u}^{(s)}$ is in $F^{(s)}$ it is on an SMP path ending at $\mathbf{q}_{1}$. Denote the part of the path that begins at $\mathbf{q}_{u}^{(s)}$ by $\left\{\mathbf{q}_{u}^{(s)}, \ldots \mathbf{q}_{1}\right\}$. The no swapping condition above (which requires GARP) ensures that this path does not contain a demand on the th expansion path. Thus we put $\mathbf{q}_{t}^{(s+1)}$ at the start of this SMP path to create a new SMP path. Thus $\mathbf{q}_{t}^{(s)} \in \bar{Q}\left(\mathbf{q}_{1}\right)$ for all $t$ implies $\mathbf{q}_{t}^{(s+1)} \in \bar{Q}\left(\mathbf{q}_{1}\right)$ for all $t$. Since $\mathbf{q}_{t}^{(1)} \in \bar{Q}\left(\mathbf{q}_{1}\right)$ for all $t$, this establishes the result.

The final part of the proof is to simply note that since our algorithm chooses points from a finite set $\bar{Q}\left(\mathbf{q}_{1}\right)$ and discards a finite number of points at each iteration (the 'monotonicity' condition), we terminate in a finite number of steps.

PART B. $\left(\mathbf{q}_{t} \geq \mathbf{q}_{t}^{B}\right) \Rightarrow\left(\mathbf{q}_{t} R \mathbf{q}_{1}\right)$ follows from the construction of $\bar{Q}\left(\mathbf{q}_{1}\right)$ in part A. To prove the converse let $\mathbf{q}_{t} R \mathbf{q}_{1}$. This requires that there be a preference ordered path that 
starts at $\mathbf{q}_{t}$ and ends at $\mathbf{q}_{1}$. Without loss of generality let this path be $\left\{\mathbf{q}_{t}, \mathbf{q}_{u}, \mathbf{q}_{v}, \mathbf{q}_{1}\right\}$ so that:

$$
\begin{aligned}
\mathbf{p}_{t}^{\prime} \mathbf{q}_{t} & \geq \mathbf{p}_{t}^{\prime} \mathbf{q}_{u} \\
\mathbf{p}_{u}^{\prime} \mathbf{q}_{u} & \geq \mathbf{p}_{u}^{\prime} \mathbf{q}_{v} \\
\mathbf{p}_{v}^{\prime} \mathbf{q}_{v} & \geq \mathbf{p}_{v}^{\prime} \mathbf{q}_{1}
\end{aligned}
$$

Recalling that we denote the $t$ th element of $Q^{B}\left(\mathbf{q}_{1}\right)$ by $\mathbf{q}_{t}^{B}$, by step 2 of the algorithm we have:

$$
\begin{aligned}
\mathbf{p}_{t}^{\prime} \mathbf{q}_{u}^{B} & \geq \mathbf{p}_{t}^{\prime} \mathbf{q}_{t}^{B} \\
\mathbf{p}_{u}^{\prime} \mathbf{q}_{v}^{B} & \geq \mathbf{p}_{u}^{\prime} \mathbf{q}_{u}^{B} \\
\mathbf{p}_{v}^{\prime} \mathbf{q}_{1} & \geq \mathbf{p}_{v}^{\prime} \mathbf{q}_{v}^{B}
\end{aligned}
$$

$>$ From (A.6) and (A.3) and weak monotonicity we have $\mathbf{q}_{v} \geq \mathbf{q}_{v}^{B}$. This and (A.2) and (A.5) gives $\mathbf{q}_{u} \geq \mathbf{q}_{u}^{B}$. From (A.4) and (A.1) we thus have $\mathbf{q}_{t} \geq \mathbf{q}_{t}^{B}$.

\section{Proof of Proposition 3.}

The proof is analogous to that for Proposition 2.

\section{Proof of Proposition 4.}

Given the budget share form of the Slutsky equation (3.8) and the additive structure in (3.9) we have by differentiating both side of (3.8) with respect to $\ln x$ then with respect to $\mathbf{z}$ gives

$$
\mathbf{m}_{z}^{k} g_{x x}^{j}=\mathbf{m}_{z}^{j} g_{x x}^{k} .
$$

If $\mathbf{m}_{z}^{k}$ and $\mathbf{m}_{z}^{j}$ are unrestricted this must hold for any values of $\mathbf{m}_{z}^{k}$ and $\mathbf{m}_{z}^{j}$. If either $\mathbf{m}_{z}^{k}$ or $\mathbf{m}_{z}^{j}$ are allowed to be zero then this implies $g_{x x}^{j}=g_{x x}^{k}=0$.

\section{Proof of Proposition 5.}

Assumptions:

A1: $\varepsilon_{j i}^{s}$ are assumed mutually independent and have finite second moments

A2: $E\left(\varepsilon_{j i}^{s} \mid \ln x, z^{s}\right)=0$

A3: $\ln x_{i}$ is independently distributed with density $\widehat{f}^{s}(\cdot)$ that is two times boundedly differentiable.

A4: $\widehat{f}^{s}(\cdot)\left(\widehat{r}^{s}(\cdot)\right)^{2}$ are two times boundedly differentiable functions.

A5: $\left(\boldsymbol{\alpha}^{\prime}, \boldsymbol{\theta}^{\prime}\right)$ is in a bounded and open set.

A6: The twice boundedly differentiable weight function $\varpi$, is non-negative and positive only on the interior of a compact interval $\Xi_{x}$. For all points $x \in \Xi_{x}$ we have that $f^{s}(\ln x)$ $>0$ and that for all $\left(\overline{\boldsymbol{\alpha}}^{\prime}, \boldsymbol{\theta}^{\prime}\right), x, z \in \Theta \times \Xi$ that $f^{0}\left(\ln x-\phi\left(\mathbf{z}^{s \prime} \boldsymbol{\theta}\right)\right)>0$.

A7: No parameter vector $\left(\boldsymbol{\alpha}^{\prime}, \boldsymbol{\theta}^{\prime}\right) \neq\left(\boldsymbol{\alpha}_{0}^{\prime}, \boldsymbol{\theta}_{0}^{\prime}\right)$ exists such that for some $j, g_{j}^{s}(\ln x)=$ $\mathbf{z}^{s \prime} \boldsymbol{\alpha}_{j}+g_{j}^{0}\left(\ln x-\phi\left(\mathbf{z}^{s \prime} \boldsymbol{\theta}\right)\right)$ almost all $x \in \Xi_{x}$. 
A8: The same kernel is used for all $s=0,1, . ., S$ groups with bandwidth $n_{s} h^{5} \longrightarrow \infty$, $n_{s} h^{6} \longrightarrow 0$ as $n_{s} \longrightarrow \infty$.

With assumptions A1 - A8 in place, Proposition 5 follows directly from Lemmas 1-6 and Theorem 1 in Pinske and Robinson (1995).

Discussion of assumptions in Proposition 5:

A1-A4 are standard assumptions and follow from the model specification in sections 3.1 and 3.2. A5 relates to the adult equivalence scale parameters for children (relative to the base case of a couple with no children). As pointed out in the text, these are bounded between the adult scale and zero. Given the boundedness of $\boldsymbol{\theta}, f^{0}(\ln x-$ $\left.\phi\left(\mathbf{z}^{s /} \boldsymbol{\theta}\right)\right)>0$ follows. A7 is guaranteed by assuming that at least one good has strictly nonlinear Engel curves (actually nonlinear relationship between the share and log total expenditure). This has been established in many empirical applications to UK data (see Banks, Blundell and Lewbel (1997), for example). A8 follows from our common choice of kernel and the bandwidth condition is satisfied under cross validation.

\section{B. Welfare Bound Algorithms}

\section{B.1. Illustration of Algorithm A.1}

Figure B.1 illustrates the algorithm ${ }^{37}$. We denote the sets $W$ and $F$ in the $n$ 'th iteration by $W_{n}, F_{n}$. In the first iteration step (1) begins with $W_{1}=\left\{\mathbf{q}_{1}, \mathbf{q}_{2}\left(x_{2}^{\prime}\right), \mathbf{q}_{3}\left(x_{3}^{\prime}\right), \overline{\mathbf{q}}_{4}\right\}$, where $x_{2}^{\prime}=\mathbf{p}_{2}^{\prime} \mathbf{q}_{1}$ and $x_{3}^{\prime}=\mathbf{p}_{3}^{\prime} \mathbf{q}_{1}$. Clearly $\overline{\mathbf{q}}_{4} P^{0} \mathbf{q}_{2}$ and hence $\overline{\mathbf{q}}_{4} P \mathbf{q}_{1}$. In step (2) $F_{1}=$ $\left\{\mathbf{q}_{1}, \mathbf{q}_{2}\left(x_{2}^{\prime}\right), \mathbf{q}_{3}\left(x_{3}^{\prime}\right), \mathbf{q}_{4}\left(x_{4}^{\prime}\right)\right\}$ since $\overline{\mathbf{q}}_{4} P_{4}^{0} \mathbf{q}\left(x_{4}^{\prime}\right)$. Because $W_{1} \neq F_{1}$ we set $W_{2}=F_{1}$ and go to step (2) at the second iteration. Now $F_{2}=\left\{\mathbf{q}_{1}, \mathbf{q}_{2}\left(x_{2}^{\prime}\right), \mathbf{q}_{3}\left(x_{3}^{\prime}\right), \mathbf{q}_{4}\left(x_{4}^{\prime}\right)\right\}$ and in step (3) the iteration ends defining $Q_{B}\left(\mathbf{q}_{1}\right)=\left\{\mathbf{q}_{1}, \mathbf{q}_{2}\left(x_{2}^{\prime}\right), \mathbf{q}_{3}\left(x_{3}^{\prime}\right), \mathbf{q}_{4}\left(x_{4}^{\prime}\right)\right\}$. Algorithm A.2 proceeds in a similar way giving $Q_{W}=\left\{\mathbf{q}_{1}, \mathbf{q}_{2}\left(x_{2}\right), \mathbf{q}_{2}\left(x_{3}\right), \mathbf{q}_{4}\left(x_{4}\right), x_{4} / p_{4}^{1}, x_{1} / p_{1}^{2}\right\}$ but A.2 has the additional step which identifies the final two points on the $q^{1}=0$ and $q^{2}=0$ axes. The dashed lines marked 'upper' and 'lower' shows the bounds on $c\left(\mathbf{p}_{z}, u\left(\mathbf{q}_{1}\right)\right)$ given by $\min \left\{\mathbf{p}_{z}^{\prime} \mathbf{q}_{t} \mid \mathbf{q}_{t} \in Q_{W}\left(\mathbf{q}_{1}\right)\right\}$ and $\min \left\{\mathbf{p}_{z}^{\prime} \mathbf{q}_{t} \mid \mathbf{q}_{t} \in Q_{P}\left(\mathbf{q}_{1}\right)\right\}$ for some new set of relative prices $\mathbf{p}_{z}$.

\section{Simulating measurement error}

Given a period $t$ total outlay $x_{t}$ we set expenditure on good $j$ in that period equal to $\gamma_{j t} x_{t}$ for $j=1,2 \ldots, J$. We choose the $\gamma_{j t}$ weights in the following way. First, we draw $\tilde{\gamma}_{j t}$ from a Beta distribution with parameters $\left(a_{j}, b_{j}\right)$ (where the distribution parameters are kept constant over time). To do this, we first have to calibrate the two parameters for each good. To fix one parameter we set $E\left(\tilde{\gamma}_{j t}\right)=\omega_{j}$ where the latter is a given budget share (see below). Given the usual expression for the mean of Beta distributed variable this implies that we must set:

$$
b_{j}=\frac{\left(1-\omega_{j}\right) a_{j}}{\omega_{j}}
$$

\footnotetext{
${ }^{37}$ We subscript the sets defined at each stage by the current iteration of the algorithms.
} 
Figure B.1: Bounds on the cost-of-living index using expansion paths

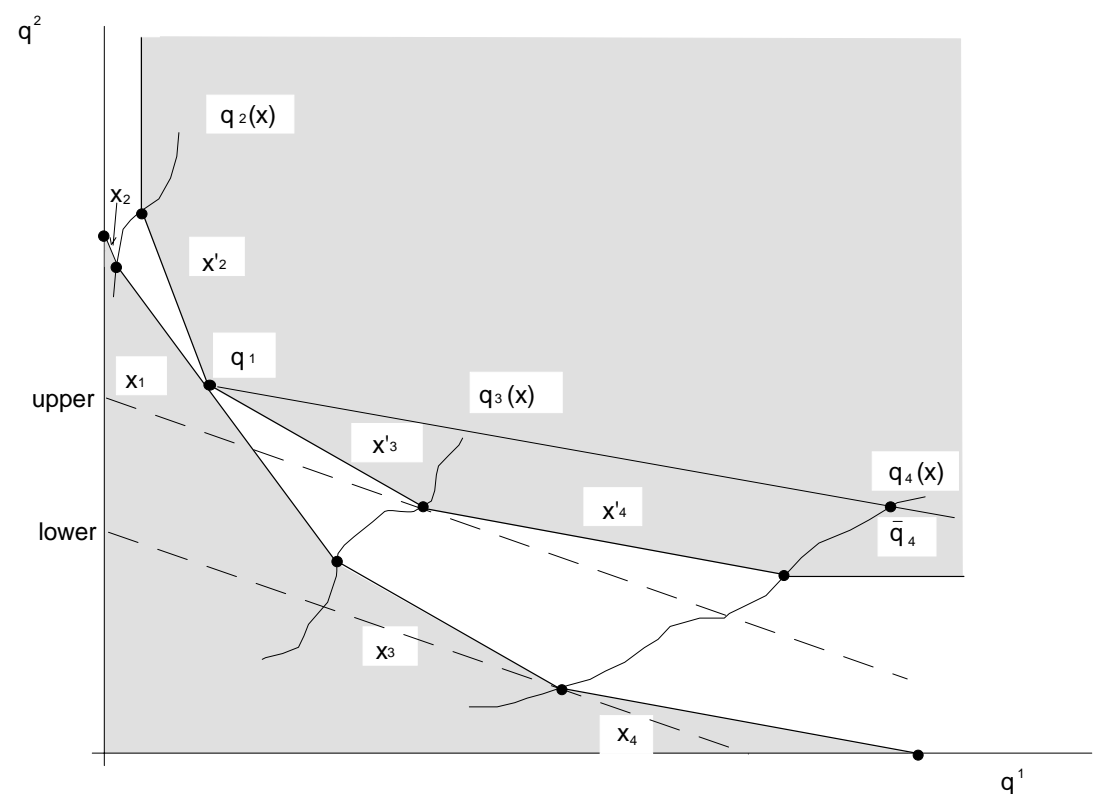


for each good $j$. It only remains to calibrate the $a_{j}$ parameters. The variance of a Beta distributed random variable is given by:

$$
\sigma_{j}^{2}=\frac{a_{j} b_{j}}{\left(1+a_{j}+b_{j}\right)\left(a_{j}+b_{i}\right)^{2}}=\frac{\left(1-\omega_{j}\right) \omega_{j}}{a_{j}+\omega_{j}}
$$

so that if we take a value for the variance the associated value of $a_{j}$ is given by:

$$
a_{j}=\left(\frac{\left(1-\omega_{j}\right) \omega_{j}}{\sigma_{j}^{2}}-1\right) \omega_{j}
$$

Thus we first choose $\left(\omega_{j}, \sigma_{j}^{2}\right)$ for each good and then calculate $\left(a_{j}, b_{j}\right)$ for $j=1,2 \ldots, J$. Given these parameters we can simulate a set of budget weights for each period $t$, $\left(\tilde{\gamma}_{1 t}, \tilde{\gamma}_{2 t}, \ldots, \tilde{\gamma}_{J t}\right)$. Since these will not normally sum to unity we set:

$$
\gamma_{j t}=\frac{\tilde{\gamma}_{j t}}{\sum_{j=1}^{J} \tilde{\gamma}_{j t}}
$$

Although the marginals of the joint distribution of the $\gamma_{j t}$ 's are now no longer a Beta distribution and the weights do not have the desirable property that $E\left(\gamma_{j t}\right)=\omega_{j}$ we do have that the mean is approximately equal to the data mean (using conventional expansion arguments on the mean of a ratio) and bounded between zero and unity, which suffices for our purposes. It only remains to choose the mean and variances discussed above. We use data from one representative year (1974) and set the budget shares $\omega_{j}$ 's equal to the mean budget shares. For the variance, we first take the variance of each budget share, denoted $\bar{\sigma}_{j}^{2}$ for good $j$. We take this to be an upper bound for noise in the measurement and then choose an attenuation factor $\rho$ to give the calibrating variance $\sigma_{j}^{2}=\rho \bar{\sigma}_{j}^{2}$ for each good. Thus an attenuation factor of unity gives the maximum noise and an attenuation factor of zero gives no noise. 


\section{Data}

Table D.1: Total nondurable nominal expenditure: Annual descriptive statistics.

\begin{tabular}{l|llllll}
\hline \hline Year & No. of Obs & Mean & Std Dev. & $\mathbf{1 0 \%}$ & $\mathbf{5 0 \%}$ & $\mathbf{9 0 \%}$ \\
\hline 1974 & 3386 & 39.11 & 17.95 & 20.41 & 35.19 & 62.93 \\
1975 & 3696 & 47.17 & 21.17 & 24.83 & 42.36 & 75.92 \\
1976 & 3553 & 52.79 & 24.20 & 27.75 & 47.23 & 84.15 \\
1977 & 3683 & 60.94 & 27.71 & 31.87 & 54.83 & 98.65 \\
1978 & 3583 & 67.84 & 31.33 & 35.34 & 60.78 & 108.76 \\
1979 & 3476 & 79.18 & 37.04 & 40.36 & 71.42 & 127.72 \\
1980 & 3717 & 92.84 & 43.07 & 47.67 & 82.77 & 152.70 \\
1981 & 4072 & 102.63 & 47.94 & 52.78 & 91.29 & 169.21 \\
1982 & 3974 & 108.89 & 50.10 & 56.83 & 98.15 & 175.15 \\
1983 & 3749 & 117.11 & 54.40 & 60.33 & 105.69 & 190.41 \\
1984 & 3755 & 124.71 & 59.71 & 62.81 & 110.22 & 206.58 \\
1985 & 3775 & 132.56 & 64.68 & 64.94 & 117.65 & 219.00 \\
1986 & 3826 & 143.35 & 71.64 & 69.35 & 126.01 & 240.79 \\
1987 & 3962 & 150.49 & 74.20 & 72.42 & 134.40 & 249.69 \\
1988 & 4003 & 163.01 & 83.09 & 75.71 & 145.68 & 274.40 \\
1989 & 4086 & 173.93 & 86.57 & 83.38 & 155.14 & 292.80 \\
1990 & 3772 & 191.01 & 95.95 & 91.15 & 169.15 & 320.19 \\
1991 & 3886 & 199.59 & 99.41 & 96.19 & 177.71 & 332.81 \\
1992 & 3999 & 205.58 & 97.29 & 101.02 & 185.86 & 339.20 \\
1993 & 3800 & 219.84 & 111.99 & 105.47 & 192.97 & 363.91 \\
\hline \hline
\end{tabular}

\section{References}

[1] Afriat, S.N. (1973), "On a System of Inequalities in Demand Analysis: An Extension of the Classical Method", International Economic Review, 14, 460-472.

[2] Afriat, S.N. (1977), The Price Index, London, Cambridge University Press.

[3] Ai, C. and X. Chen (2000), "Efficient Estimation of Models with Conditional Moment Restrictions Containing Unknown Functions", LSE Working Paper in Econometrics, June.

[4] Banks, J. Blundell, R.W. and Lewbel, A. (1996), "Tax Reform and Welfare Measurement: do we need Demand System Estimation?", Economic Journal, 106, 12271241. 
[5] Banks, J. Blundell, R.W. and Lewbel, A. (1997), "Quadratic Engel Curves, Indirect Tax Reform and Welfare Measurement", Review of Economics and Statistics, LXXIX, 4, 527-539.

[6] Becker, G. S. (1962), "Irrational behaviour and economic theory", Journal of Political Economy, 70, 1-13

[7] Blundell, R., Chen, X. and D. Kristensen, "Semiparametric Shape Invariant Engel Curves with Endogenous Expenditure", mimeo, University College London, November.

[8] Blundell, R. and Duncan, A. (1998) "Kernel Regression in Empirical Microeconomics" Journal of Human Resources, 33, 62-87.

[9] Blundell, R. Duncan, A. and Pendakur, K.(1998), "Semiparametric Estimation and Consumer Demand", Journal of Applied Econometrics, 13, 435-461.

[10] Bronars, S.G. (1995), "The Power of Nonparametric Tests of Preference Maximisation", Econometrica, 55, 693-698.

[11] Brown, D.J. and Matzkin, R.L. (1995), "Estimation of nonparametric functions in simultaneous models with an application to consumer demand", mimeo, Northwestern University.

[12] Brown, B.W. and Walker, M.B., (1989), "The random utility hypothesis and inference in demand systems" Econometrica, 57, 815-829.

[13] Deaton, A.S. (1983), Demand Analysis, in Handbook of Econometrics,(eds Z. Griliches and M. Intrilligator), JAI Press.

[14] Deaton A. S. and J. Muellbauer (1980), "An Almost Ideal Demand System", American Economic Review, 70, 312-36.

[15] Diewert, W.E. (1973), "Afriat and Revealed Preference Theory", Review of Economic Studies, 40, 419-426.

[16] Diewert, W.E. (1976), "Exact and Superlative Index Numbers", Journal of Econometrics, 4, 115-145.

[17] Diewert, W.E. (1981), "The Economic Theory of Index Numbers", in Essays in the Theory and Measurement of Consumer Behaviour in Honour of Sir Richard Stone, ed. by Angus Deaton, Cambridge: Cambridge University Press.

[18] Diewert, W.E. and Parkin, C. (1985), "Tests for the Consistency of Consumer Data", Journal of Econometrics, 30, 127-148.

[19] Famulari, M. (1995), "A Household-based, Nonparametric Test of Demand Theory", Review of Economics and Statistics, 77, 372-382.

[20] Fan, J. (1992), "Design Adaptive Nonparametric Regression", Journal of the American Statistical Association, 87, 998-1004. 
[21] Gorman, W. M. (1953), "Community Preference Fields", Econometrica, 21, 63-80

[22] Gorman, W. M. (1981), "Some Engel Curves", in Essays in the Theory and Measurement of Consumer Behaviour in Honour of Sir Richard Stone, ed. by Angus Deaton, Cambridge: Cambridge University Press.

[23] Grandmont, J.M. (1992), "Transformation of the Commodity Space, Behavioral Heterogeneity, and the Aggregation Problem", Journal of Economic Theory, 57, $1-35$.

[24] Härdle W. and Jerison, M. (1988), "The Evolution of Engel Curves over time", Discussion paper no. A-178. SFB 303, University of Bonn.

[25] Härdle W. (1990) Applied Nonparametric Regression, Cambridge: Cambridge University Press.

[26] Härdle W. and Linton, O. (1995), "Nonparametric Regression analysis" in Engle and McFadden, Handbook of Econometrics Vol IV, North-Holland.

[27] Härdle W. and Marron J. (1990), "Semiparametric Comparison of Regression Curves," Annals of Statistics, 18, 63-89.

[28] Hausman, J.A. and Newey, W.K. (1995), "Nonparametric Estimation of Exact Consumer Surplus and Deadweight Loss", Econometrica 63, 1445-1476.

[29] Heckman, J.J. (1974), "Effect of Child-Care Programs on Women's Work Effort", Journal of Political Economy.

[30] Hildenbrand, W. (1994), Market Demand: Theory and Empirical Evidence, Princeton: Princeton University Press.

[31] Holly, A. (1982), "A Remark on Hausman's Specification Test", Econometrica, 50: $749-59$.

[32] Houthakker, H.S. (1950), "Revealed Preference and the Utility Function", Economica, 17, 159-74.

[33] Jorgenson,D.W., L.J.Lau, and T.M.Stoker (1982), "The Transcendental Logarithmic Model of Aggregate Consumer Behavior", in Advances in Econometrics, Vol1, ed. by R. Basmann and G. Rhodes, Greenwich, Connecticut: JAI Press.

[34] Konüs, A. A. (1924), "The Problem of the True Index of the Cost of Living", reprinted in Econometrica, 7, 10-29.

[35] Koo, A.Y.C. (1963), "An Empirical Test of Revealed Preference Theory", Econometrica, 31, 646-664.

[36] Lerner, A.P. (1935-36), "A Note on the Theory of Price Index Numbers", Review of Economic Studies, 3, 50-56.

[37] Leser, C. E. V. (1963), "Forms of Engel Functions", Econometrica, 31, 694-703. 
[38] Lewbel, A. (1991), "The rank of demand systems: theory and nonparametric estimation", Econometrica, 59, 711-730.

[39] Lewbel, A (1996), "Demand systems with and without errors: reconciling econometric, random utility and GARP models", mimeo, Brandeis University, September 1996.

[40] Manser, M.E. and McDonald, R.J. (1988),"An Analysis of Substitution Bias in Measuring Inflation, 1959-1985”, Econometrica, 56, 909-930.

[41] Mattie, A. (1994), "La Coherence des Choix des Cosommateurs Suisses", Revue Suisse d'Economie Politique et de Statistique, 130, 3-20.

[42] McElroy, M.B. (1987), "Additive General Error Models for Production, Cost and Derived Demand or Share Equations" Journal of Political Economy, 95, 737-757.

[43] Mossin, A. (1972), "A Mean Demand Function and Individual Demand Functions Confronted with the Weak and Strong Axioms of Revealed Preference", Econometrica, 40, 177-192.

[44] Muellbauer, J. (1976), "Community Preferences and the Representative Consumer", Econometrica, 44, 525-543.

[45] Newey, W. Powell, J. and Vella, F. (1999), "Nonparametric Estimation of Triangular Simultaneous Equations Models", Econometrica, 67, 565-604.

[46] Pendakur, K. (1998), "Semiparametric Estimates and Tests of Base-independent Equivalence Scales", Journal Of Econometrics, 88, 1-40.

[47] Pinske, C. and Robinson, P. (1995), "Pooling Nonparametric Estimates of Regression Functions with a Similar Shape", Advances in Econometrics and Quantitative Economics, eds. G. Maddala, P. Phillips and T.N. Srinivisan, 172-195.

[48] Pollak,R.A. (1971), "The Theory of the Cost of Living Index", Research Discussion Paper No. 11, Bureau of Labor Statistics, Washington, D.C.

[49] Robinson, P.M. (1988), "Root n-consistent semiparametric regression", Econometrica, 56, 931-954.

[50] Russell, R.R. (1992), "Remarks on the Power of Nonparametric tests of ConsumerTheory Hypotheses", in L. Phlips and L.D.Taylor (eds), Aggregation, Consumption and Trade, Dordrecht: Kluwer Academic Publishes, 121-135.

[51] Samuelson, P.A. (1938), "A Note on the Pure Theory of Consumer Behavior", Econometrica, 5, 61-71.

[52] Silverman, B.W. (1986), Density Estimation for Statistics and Data Analysis, London: Chapman and Hall.

[53] Sippel, R. (1997), "An Experiment on the Pure Theory of Consumer's Behaviour", Economic Journal, 107, 1431-1444. 
[54] Varian, H.(1982), "The Nonparametric Approach to Demand Analysis ", Econometrica, 50, 945-974.

[55] Varian, H.(1983), "Nonparametric Tests of Consumer Behaviour", Review of Economic Studies, 99-110.

[56] Varian, H.(1986), "Nonparametric Analysis of Optimizing Behavior with Measurement Error", Journal of Econometrics30, 445-459.

[57] Working, H., (1943), "Statistical Laws of Family Expenditure", Journal of the American Statistical Association, 38, 43-56. 